\title{
15. Cardiopatías congénitas
}

\subsection{Válvula aórtica cuadricúspide: una anomalía congénita rara}

Chih Lin Chen, Hernández Del Rio Jorge Estuardo

Hospital Civil Fray Antonio Alcalde.

Tipo de estudio: Reporte de casos clínicos con revisión de fuentes

Introducción: La válvula aórtica cuadricúspide es una anomalía extremadamente rara con reporte del primer caso en 1862. Este defecto tiene una frecuencia $<0.05 \%$. Sin embargo, un estudio realizado en Mayo Clinic reporta una frecuencia de $0.006 \%$. Aquí presentamos a un paciente con válvula aórtica cuadricúspide. Descripción del caso: Paciente masculino de 14 años sin comorbilidades ni antecedentes familiares; es referido de medicina interna para abordaje de síndrome edematoso y diarrea crónica de un mes de evolución. Al examen

Figura 15.1.1.
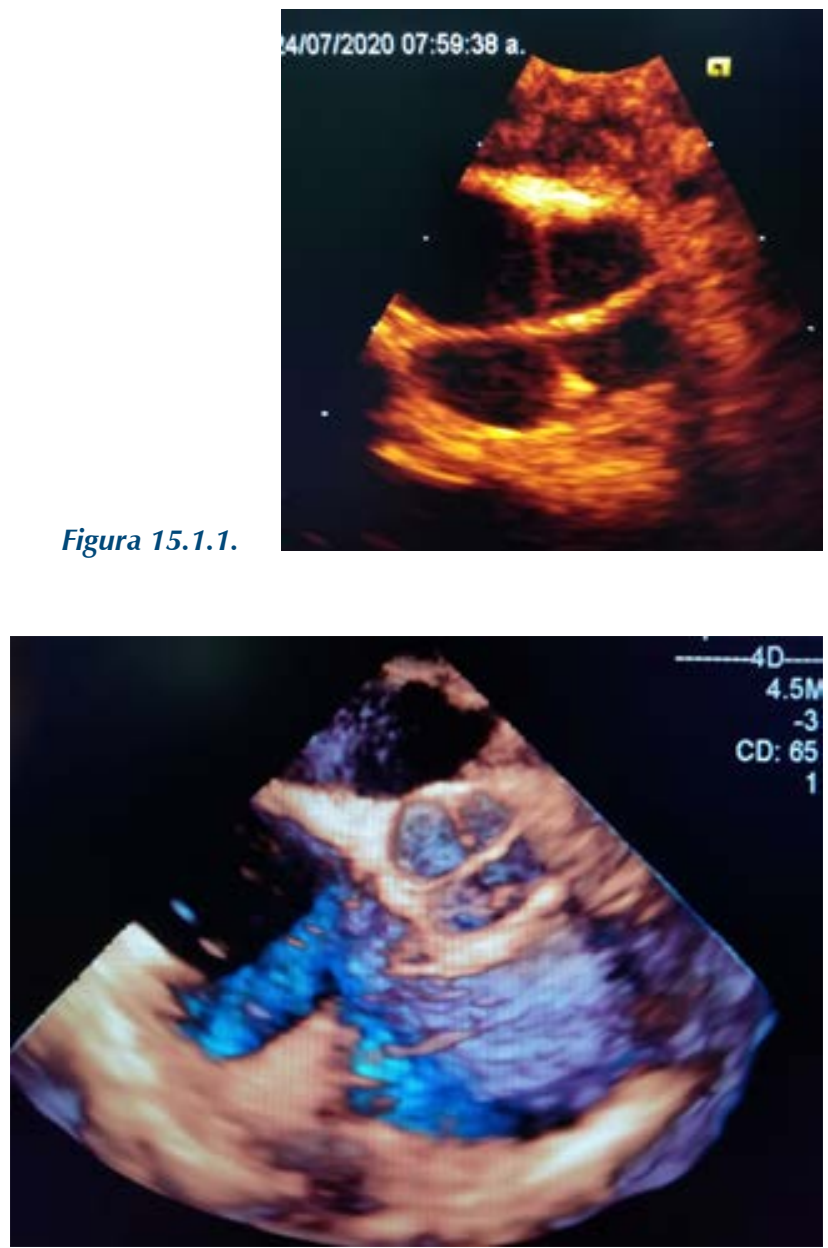

Figura 15.1.2. físico se encuentra con signos vitales normales. Se realiza ecocardiograma transesofágico en el cual se aprecia de forma incidental cuatro cúspides aórticas con insuficiencia leve y función ventricular normal. Metodología: Se realiza una búsqueda de artículos relacionados a válvula aórtica cuadricúspide en Google, PubMed y Elsevier. Se utilizaron las palabras válvula aórtica cuadricúspide y quadricuspid aortic valve. Resultados: La válvula aórtica cuadricúspide es una anomalía congénita rara con una frecuencia de $0.006 \%$ de acuerdo con un estudio realizado en Mayo Clinic. El estado funcional de la válvula aórtica cuadricúspide de los pacientes revisados se encuentra con predominio de insuficiencia. Es una enfermedad con progresión lenta y baja mortalidad a lo largo de 10 años, aun con cirugía. Discusión: En 1973, Hurwitz y Roberts propusieron una clasificación en siete subtipos de A-G dependiendo del tamaño de las valvas, los grupos A-C se presentan con mayor frecuencia. Nuestro paciente pertenece al grupo E y, según la revisión, es una enfermedad de baja mortalidad. Se dará seguimiento al paciente a lo largo del año.

\subsection{Divertículo ventricular derecho simulando tumor cardiaco}

Rodríguez López Sergio Alberto, Germán Arroyo Christopher,

García Dávalos Israel, Salazar Lizárraga David, Márquez González Horacio, López Gallegos Diana, Yáñez Gutiérrez Lucelli

Hospital de Cardiología del Centro Médico Nacional

Siglo XXI, Instituto Mexicano del Seguro Social.

Tipo de estudio: Prueba diagnóstica

Introducción: El divertículo ventricular (DV) derecho es una entidad congénita rara definida como un saco o bolsa que se extiende desde la pared ventricular; tiene un curso benigno, pero puede simular otras

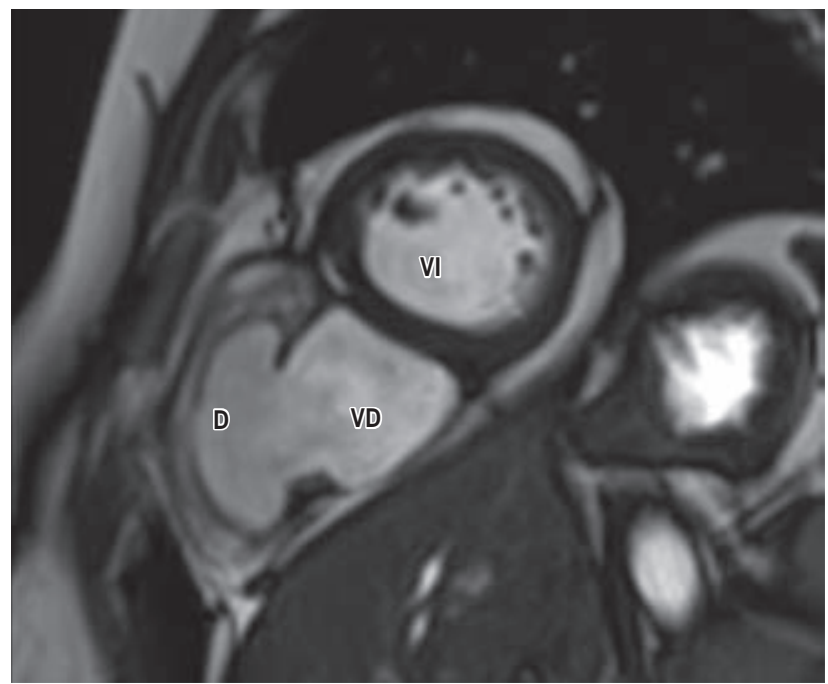

Figura 15.2.1. 


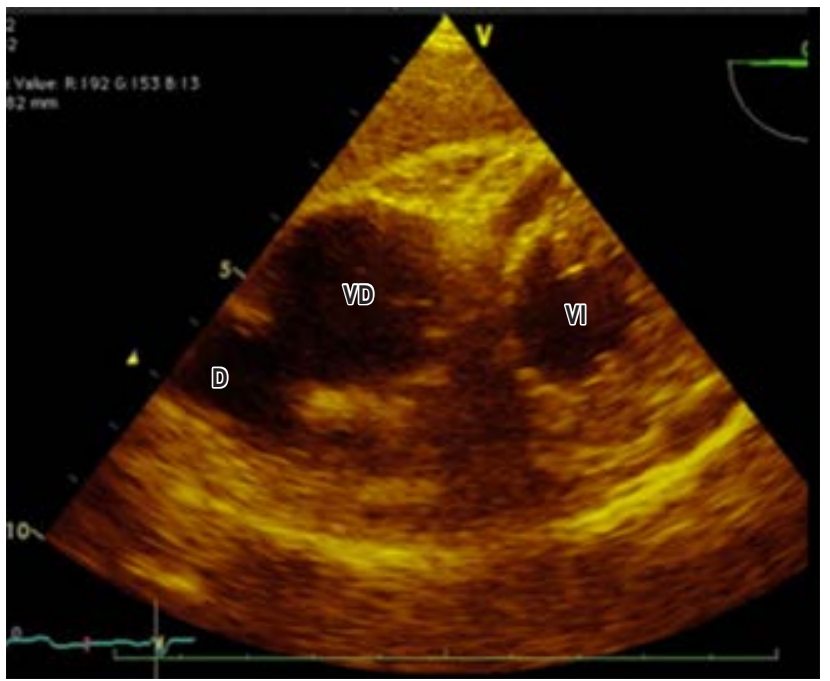

Figura 15.2.2. patologías que llevan a historias naturales distintas. Descripción del caso: Femenino de 28 años, sin antecedentes. Inicia su padecimiento tras revisión médica de rutina con presencia de soplo cardiaco, motivo por el que es referida a nuestra unidad. Al realizar ecocardiograma se observa imagen hiperecoica a nivel del ventrículo derecho, así como estenosis pulmonar ligera, por lo cual se sospecha de tumor cardiaco. Metodología: A través del método booleano se realizó búsqueda de términos $\mathrm{MeSH}$ «right ventricle diverticulum» en las bases de datos con repositorios de evidencia científica arbitrada indizada (PubMed) y no indizadas (Google Scholar). Resultados: La incidencia de DV derecho es baja, en un estudio por Srichai se encontró DV izquierdo en $2.2 \%$ de 675 pacientes sin encontrar DV derecho. Takahiko y colaboradores encontraron DV derecho en dos de 324 pacientes (0.6\%). La contracción simultánea del divertículo junto con la pared libre del ventrículo es útil para diferenciar del aneurisma ventricular. Discusión y conclusiones: Se solicita resonancia magnética con gadolinio en la cual se observa protrusión cardiaca derivada de la pared lateral del ventrículo derecho, con patrón contráctil, sin evidencia de reforzamiento tardío. Se concluye como divertículo del ventrículo derecho. Se decide no ofrecer tratamiento quirúrgico debido al curso benigno de esta entidad.
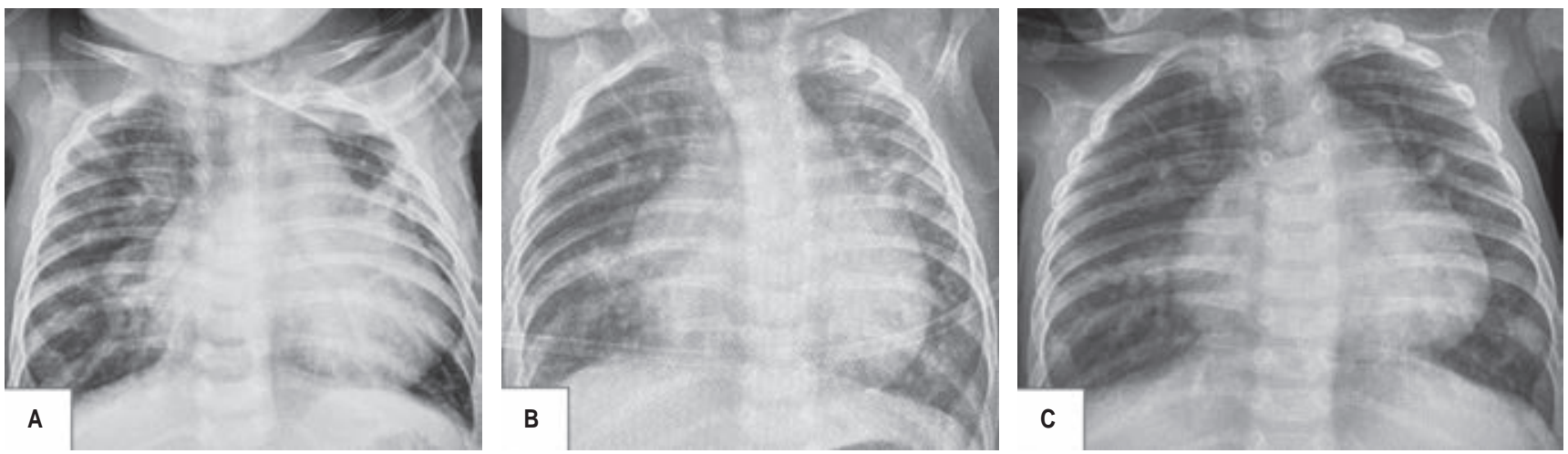

Figura 15.3.1: Radiografía de tórax. A) Durante dificultad respiratoria. B) A las 48 horas postextubación. C) Un mes después de su egreso.
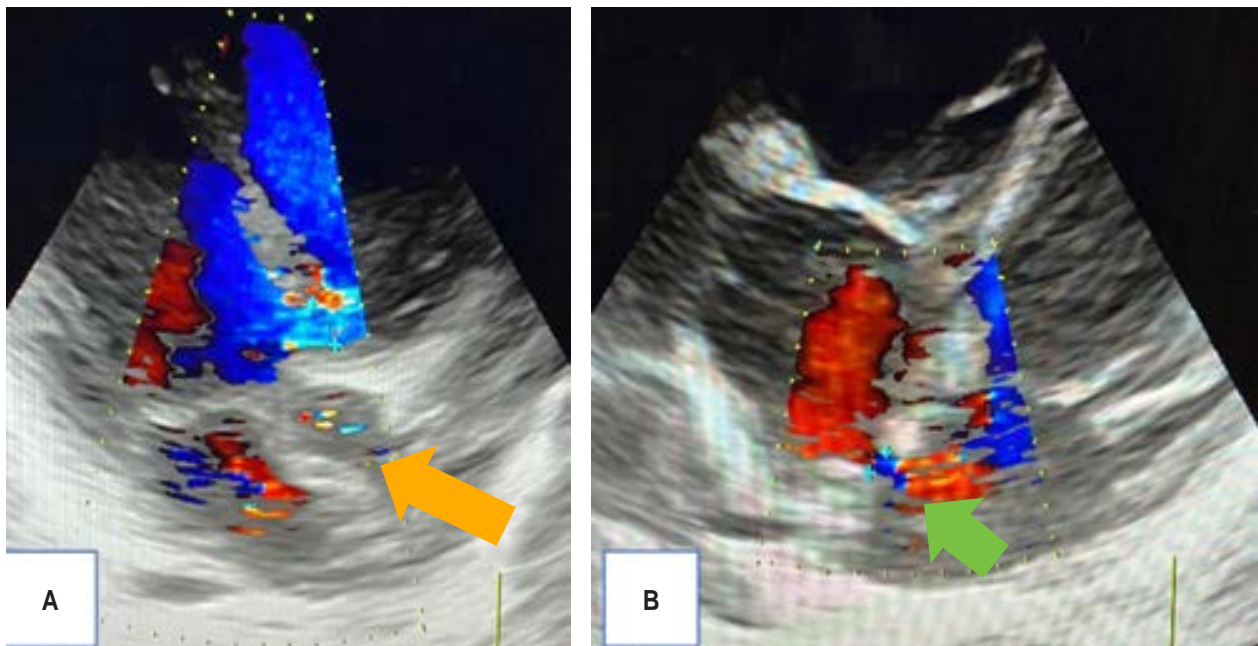

Figura 15.3.2:

Ecocardiograma que muestra los defectos cardiacos. A) Eje apical cinco cámaras con comunicación interventricular subaórtica (flecha amarilla). B) Eje subcostal con comunicación interauricular tipo ostium secundum (flecha verde). 


\subsection{Neumonía por SARS-CoV-2 en lactante cardiópata con resolución favorable}

Cordero Oropeza Alejandro Ismael, Delgado Rodríguez Ricardo Jael, Villanueva López Noé, Ramírez González Magaly, Vargas García Nadia, Rangel González Moisés Fernando, Lira Hidalgo Agustín Instituto Mexicano del Seguro Social.

Tipo de estudio: Reporte de casos clínicos con revisión de fuentes

Introducción: La infección por COVID-19, causante del síndrome respiratorio agudo severo (SARS-CoV-2), es un betacoronavirus descrito en un grupo de pacientes que presentaron síntomas de neumonía en Wuhan, China. En este país, de 72,314 pacientes, menos del 1\% correspondía a niños de entre uno y 18 años, de los cuales 731 fueron confirmados positivos con PCR y de éstos ninguno tuvo cardiopatía congénita. Descripción del caso: Femenino de seis meses, con neumopatía, hipotiroidismo congénito, CIA ostium secundum, CIV subaórtica; manejada con levotiroxina, captopril, espironolactona y furosemida. La paciente es hospitalizada por diarrea, al tercer día presenta polipnea y dificultad respiratoria, se agrega fiebre y deterioro del patrón respiratorio; intubación orotraqueal por 48 horas. Cursó con linfopenia, aumentó PCR, radiografía con infiltrados broncoalveolares, prueba para COVID-19 positiva. Seguimiento a un mes: asintomática respiratoria. Metodología: Se realizó búsqueda bibliográfica mediante Cochrane de los últimos cuatro meses, con el mayor número de muestras hasta el momento. Resultados: Si bien estamos ante la situación de reciente conocimiento sobre el comportamiento clínico de la patología respiratoria por SARS-CoV-2 en pacientes pediátricos sin comorbilidades, resulta de interés conocer la evolución de estos pacientes asociada a diferentes comorbilidades. Nuestra paciente tuvo resolución favorable a pesar de las patologías de base; en el futuro mediato será más frecuente esta patología con múltiples comorbilidades. Discusión: En una serie de casos tan grande como la reportada en Wuhan, China, no reportaron un solo paciente con características semejantes a la nuestra; por lo que esperamos que el reporte de este caso sirva para series más grandes en poblaciones de niños mexicanos. Se dará seguimiento por el riesgo inherente de miocarditis viral.

\subsection{Daño miocárdico severo secundario a síndrome coronario agudo por enfermedad de Kawasaki}

Núñez Méndez José Gilberto, Núñez-Méndez Rodrigo, Germán-

Arroyo Cristopher, García-Dávalos Israel, Márquez-González Horacio, Yáñez-Gutiérrez Lucelli, Almeida-Gutiérrez Eduardo

Hospital de Cardiología del Centro Médico Nacional Siglo XXI.

Tipo de estudio: Reporte de casos clínicos con revisión de fuentes

Introducción: La enfermedad de Kawasaki es la primera causa de enfermedad cardiaca adquirida en la edad pediátrica. El 25\% de los pacientes no tratados con inmunoglobulina intravenosa presentarán dilatación aneurismática de las arterias coronarias y 5\% sufrirán dicha complicación a pesar del tratamiento. Descripción del caso: Masculino de nueve años. Presentó fiebre, exantema y faringoamigdalitis dos años previos. Disnea de medianos esfuerzos. Electrocardiograma: infarto en cara anterior. Ecocardiograma: ventrículo izquierdo dilatado, DDVI 59 mm, FEVI 30\%, SLG -8\% y acinesia anterior. Angiotomografía coronaria: circunfleja ectásica, dilatación aneurismática proximal de la descendente anterior y oclusión total crónica en segmento medio. No fue tributario a intervención coronaria percutánea. Metodología: Se realizó una
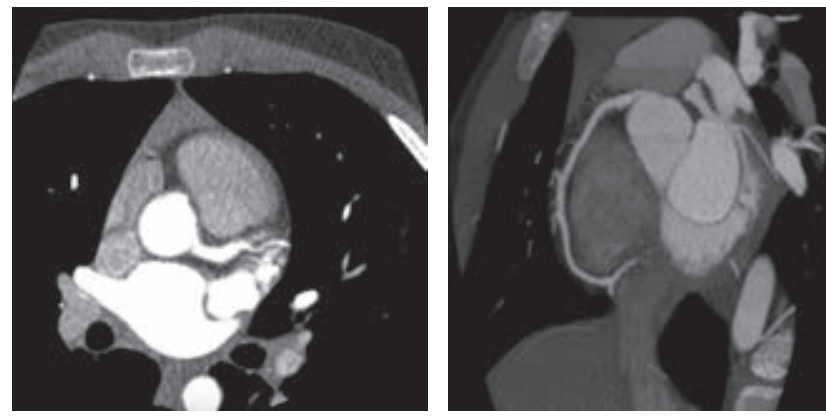

Figura 15.4.1: Angiotomografía coronaria: demostró una circunfleja ectásica (score Z 3.97), dilatación aneurismática proximal de la descendente anterior (Z 5.69) y una oclusión total crónica en el segmento medio.
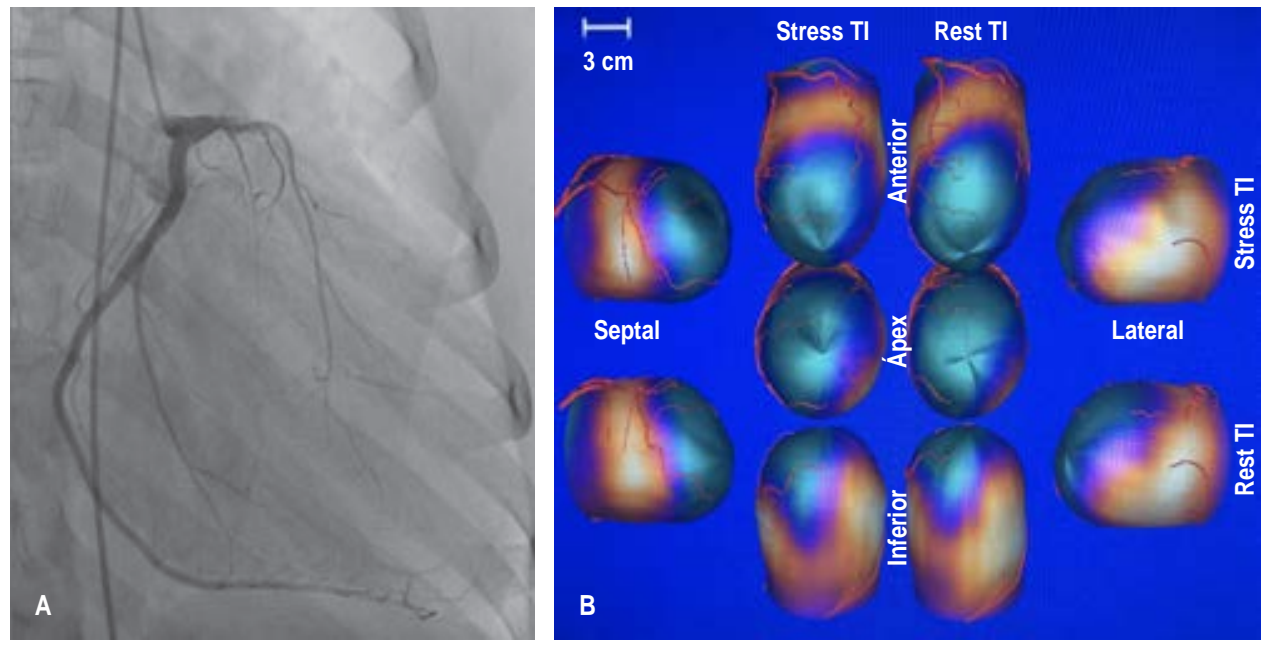

Figura 15.4.2:

A) Angiografía coronaria, proyección oblicua anterior derecha. B) Tomografía de emisión de fotón único, reconstrucción tridimensional. 
búsqueda binaria en bases de datos de revistas indexadas con factor de impacto, PubMed y Cochrane, y en bases de datos de publicaciones arbitradas sin factor de impacto, Google Scholar. Se eligieron los artículos de revisión, guías de práctica clínica y series de casos que mostraron información relacionada con la situación de nuestro paciente. Resultados: Este caso ejemplifica las secuelas cardiovasculares de la enfermedad de Kawasaki, con alta sospecha diagnóstica por dilatación aneurismática en los segmentos proximales del sistema coronario izquierdo y el antecedente de cuadro febril y enfermedad exantemática. El infarto miocárdico es la complicación cardiovascular más frecuente y el cuadro clínico puede ser atípico, como en el caso de nuestro paciente. Discusión: La enfermedad de Kawasaki es un diagnóstico clínico y no existen pruebas confirmatorias específicas, el diagnóstico siempre debe ser considerado en el paciente pediátrico con infarto agudo de miocardio y dilatación coronaria.

\subsection{Diferencias en el strain en cardiopatías congénitas con fisiología univentricular}

Cordero Oropeza Alejandro Ismael,

Liévano Cruz Roger Alexis De Jesús, Noriega Flores Elvira Guadalupe, López Gallegos Diana, García Dávalos Israel, Salazar Lizárraga David, Márquez Congénito Horacio

Instituto Mexicano del Seguro Social.

Tipo de estudio: Registros locales o nacionales

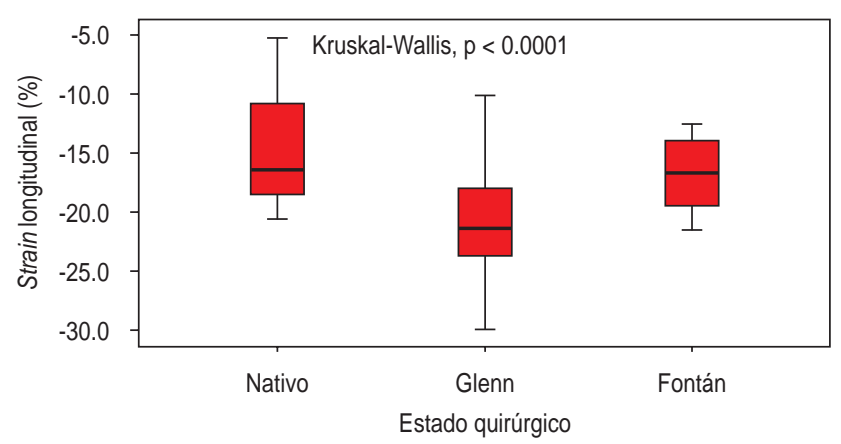

Figura 15.5.1: Diferencias del valor del strain longitudinal según el estado postquirúrgico de los pacientes con fisiología univentricular.

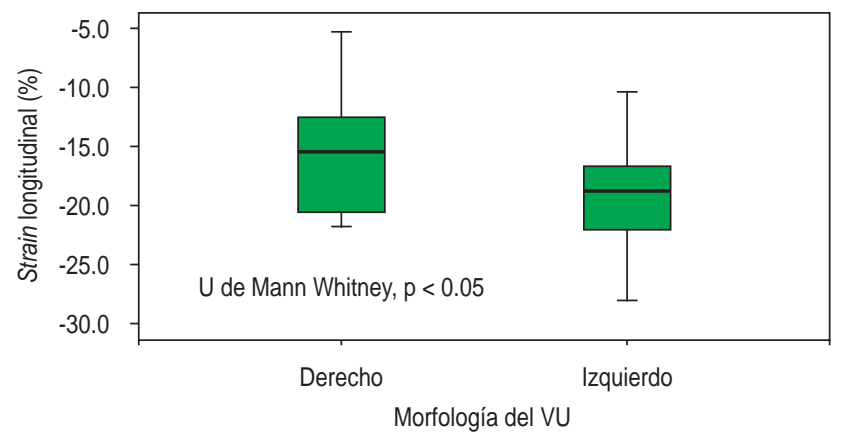

Figura 15.5.2: Grado de deformación según la morfología del ventrículo único.
Introducción: Se conoce el comportamiento de los valores del strain en corazones estructuralmente sanos, pero se sabe poco de estos parámetros en pacientes con fisiología univentricular, por lo cual el hueco en el conocimiento radica en conocer las diferencias cuantitativas entre las distintas fases correctivas de las cardiopatías con fisiología univentricular, debido a que estas poblaciones son limitadas en múltiples centros hospitalarios. Objetivo: Determinar la diferencia cuantitativa del strain entre los pacientes reparados con cirugía cavopulmonar versus sin reparación (estado nativo de la cardiopatía o reciente diagnóstico). Metodología: Mediante ecocardiograma transtorácico se procesó una imagen en cuatro cámaras para la obtención del strain longitudinal. Se realizaron pruebas de comparación con estadística de muestras no relacionadas (Kruskal-Wallis) y se utilizaron en las variables cuantitativas prueba U de Mann-Whitney. Resultados: El strain longitudinal mejora con cualquiera de los dos procedimientos quirúrgicos paliativos (Glenn y Fontan); se observó que la morfología del ventrículo único influye sobre el strain teniendo para ello que en pacientes con ventrículo sistémico de morfología izquierda la mediana del valor del strain es de $-19 \%$ y para el ventrículo sistémico de morfología derecha se conserva en -15\%. Discusión y conclusiones: El rango del valor del strain longitudinal en los pacientes con cardiopatías univentriculares nativas es muy amplio y se incrementa aún más en pacientes con Glenn vs Fontan.

\subsection{Síndrome coronario agudo en paciente con transposición congénitamente corregida de grandes vasos}

Macossay Santiago Sigifredo, Padilla Gómez Bulmaro Irving, Germán Arroyo Cristopher, García Dávalos Israel, Salazar Lizárraga David, Márquez González Horacio, Almeida Gutiérrez Eduardo

UMAE Hospital de Cardiología del Centro Médico Nacional Siglo XXI.

Tipo de estudio: Reporte de casos clínicos con revisión de fuentes

Introducción: La transposición congénitamente corregida de grandes vasos (TCCGV) es una condición muy rara caracterizada por discordancia atrioventricular y ventrículo arterial. Representa $0.05 \%$ de todas las anomalías congénitas. Existen pocos reportes previos de pacientes con TCCGV y síndrome coronario agudo. Descripción del caso: Hombre de 51 años, antecedentes: TCCGV, comunicación interventricular subaórtica y dextrocardia. Acude por dolor torácico. El electrocardiograma muestra bloqueo-fascicularanterior solamente, sin elevación del ST. Incremento de troponina T. Ecocardiograma: situs inversus, doble discordancia, fracción de expulsión $40 \%$, hipocinesia generalizada, comunicación interventricular subaórtica. Estudio de perfusión: infarto anteroseptal e inferior con isquemia residual moderada. Cateterismo: coronarias sin lesiones significativas. Metodología: A través del método booleano se realizó búsqueda de términos $\mathrm{MeSH}$ «congenitally corrected transposition of great arteries» [and] «myocardial infarction» en las bases de datos con repositorios de evidencia científica arbitrada indizada (PubMed) y no indizadas (Google Scholar). Resultados: En la TCCGV la coronaria derecha irriga al ventrículo derecho que se encarga de la circulación sistémica y se dilata o presenta hipertrofia; con esto, el aporte de sangre resulta insuficiente con desequilibrio oferta-demanda de oxígeno causando isquemia. Resulta un caso interesante debido a que el síndrome coronario agudo no es común en la TCCGV. Discusión: Este caso muestra la presentación típica de un síndrome coronario agudo sin elevación del segmento ST en 

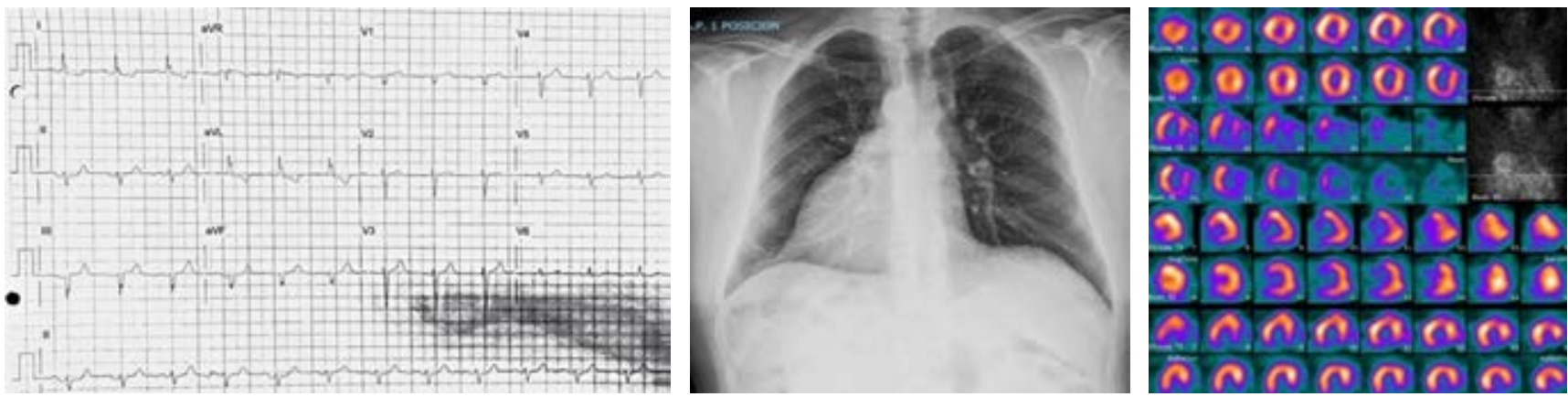

Figura 15.6.1.
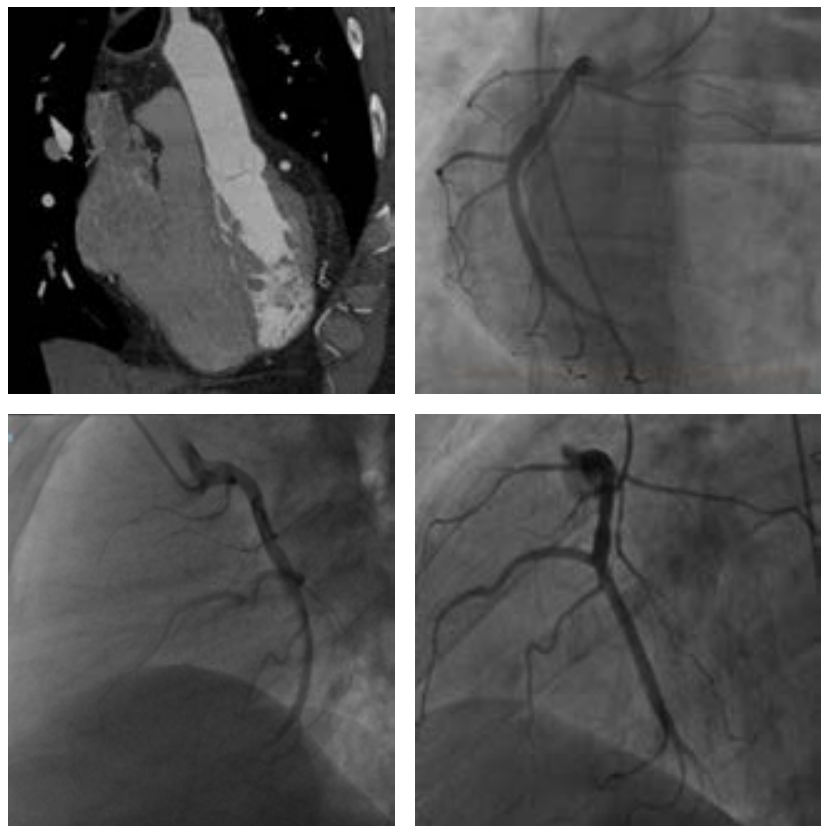

Figura 15.6.2.

el contexto de una cardiopatía congénita compleja. Se estratifica con estudio de perfusión y posteriormente se realiza coronariografía sin lesiones coronarias, por lo que se concluye infarto del miocardio tipo 2 .

\subsection{Origen anómalo de la arteria circunfleja en asociación con membrana subaórtica}

Arriaga Torres Martín José Roberto

Hospital de Cardiología del Centro Médico Nacional Siglo XXI.

Tipo de estudio: Reporte de casos clínicos con revisión de fuentes

Introducción: Las anomalías congénitas coronarias se presentan de 0.2 a $1.3 \%$ basado en series angiográficas en adultos. La más frecuente es el origen anómalo de la circunfleja (0.37 a $0.7 \%$ ), nace del seno coronario derecho o de la porción proximal de la arteria coronaria derecha. La relación del trayecto arterial con la
Figura 15.7.1.

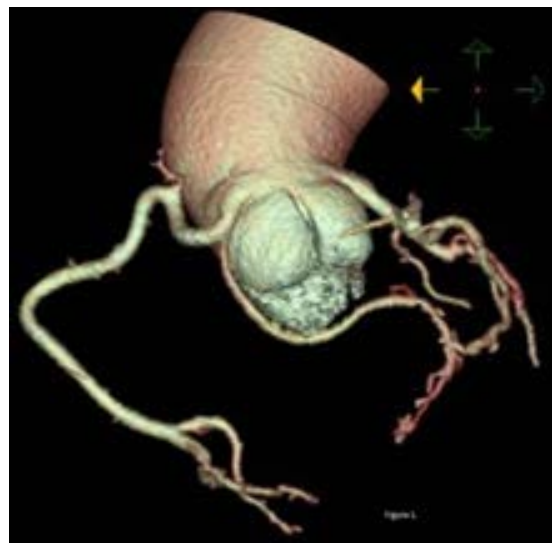

aorta y la arteria pulmonar determinan el pronóstico. Descripción del caso: Hombre de 46 años; disnea progresiva hace un año, dolor torácico opresivo cinco minutos, remisión espontánea. Soplo mesotelesistólico II/IV, escape aórtico, Landolfi positivo, retumbo de Austin Flint. Desviación izquierda del QRS. Ecocardiograma: membrana subaórtica $6 \mathrm{~mm}$, doble lesión aórtica severa. Angiotomografía: membrana en continuidad mitroaórtica y porción septal del TSVI; circunfleja nace en la coronaria derecha proximal, con trayecto retroaórtico. Metodología: A través del método booleano se realizó búsqueda de términos $\mathrm{MeSH}$ «anomalous coronary origin» [and] «circumflex branch» [and] «congenital disorders» en las bases de datos con repositorios de evidencia científica arbitrada indizada (PubMed) y no indizada (Google Scholar). Resultados: El origen anómalo de la circunfleja con origen en la arteria coronaria derecha y trayecto retroaórtico se ha considerado como benigna; sin embargo, en asociación a estenosis subaórtica condiciona isquemia secundaria por compresión por la dilatación de la raíz aórtica. Las anomalías coronarias deben ser consideradas como un grupo diverso de alteraciones congénitas con diversas manifestaciones clínicas y mecanismos fisiopatológicos. Discusión: El origen anómalo arterial coronario es una patología poco prevalente con múltiples mecanismos fisiopatológicos y diferentes determinantes pronósticos. El origen anómalo de la arteria circunfleja desde la coronaria derecha con trayecto retroaórtico al presentarse en asociación a una cardiopatía que induce dilatación de la raíz aórtica se puede acompañar de síntomas de isquemia miocárdica. 


\subsection{Disincronía atrio ventricular y falla ventricular izquierda severa como complicación quirúrgica}

Guerrero Castillo Diego Alejandro,*

Berber Patiño Salvador, ${ }^{\ddagger}$ Germán Arroyo Christopher, ${ }^{\ddagger}$

García Dávalos Israel, ${ }^{\ddagger}$ Salazar Lizárraga David, ${ }^{\ddagger}$ Márquez González Horacio, ${ }^{\ddagger}$ Yáñez Gutiérrez Lucelli

* Hospital de Cardiología del Centro Médico Nacional

Siglo XXI. ${ }^{\ddagger}$ Instituto Mexicano del Seguro Social.

Tipo de estudio: Reporte de casos clínicos con revisión de fuentes

Introducción: El bloqueo auriculoventricular completo (BAVC) ocurre en la corrección de cardiopatías congénitas entre 1-3\%, mientras que el bloqueo tardío sucede en $0.3-0.7 \%$. Se resuelve espontáneamente de 43 a 92\%. Los mecanismos son fibrosis progresiva y esclerosis lenta a través de las vías de conducción. La presentación clínica va desde asintomáticos hasta muerte súbita por arritmias ventriculares. Descripción del caso: Femenino, cierre de CIV a los cuatro años, presentó BAVC sin bajo gasto. Colocación valvular aórtica y mitral mecánica a los 11 años. Presentó síncope a los 12 años, desarrolló fibrilación-ventricular, se desfibriló 200J, posterior taquicardia ventricular monomorfa con pulso, se cardiovirtió, salió a BAVC con QT prolongado. Se colocó marcapaso bicameral DDDR. El ecocardiograma demostró disfunción sisto-diastólica ventricular izquierda. Metodología: A través del método booleano se realizó búsqueda de términos $\mathrm{MeSH}$ «postoperative heart block», «bradycardia-induced ventricular arrhythmias» [and] «treatment» en las bases de datos con repositorios de evidencia científica arbitrada indizada (PubMed) y no indizadas (Google Scholar). Resultados:
Marañón y colegas reportaron que $4 \%$ de los pacientes con BAVC presentaron FV/TV. El sustrato propuesto es el remodelado eléctrico por la reducción de corrientes IKS e IKR. Se recomienda el implante de marcapaso con un periodo de espera $>72$ horas en el postquirúrgico. Discusión: El implante de marcapaso evita el remodelado eléctrico previniendo arritmias ventriculares y disfunción ventricular.

\subsection{Estenosis pulmonar periférica difusa como causa de hipertensión arterial pulmonar}

Rincón Gómez Imelda, García Dávalos Israel, Germán Arroyo Cristopher, López Gallegos Diana, Márquez González Horacio, Salazar Lizárraga David, Yáñez Gutiérrez Lucelli

Hospital de Cardiología del Centro Médico Nacional Siglo XXI.

Tipo de estudio: Reporte de casos clínicos con revisión de fuentes

Introducción: La estenosis arterial pulmonar periférica es poco reconocida en la población adulta, puede provocar hipertensión pulmonar, pero es probable que se diagnostique erróneamente como hipertensión arterial pulmonar idiopática o hipertensión pulmonar tromboembólica crónica. La estenosis arterial pulmonar periférica es una forma rara de cardiopatía congénita frecuentemente asociada con los síndromes de Williams y Alagille. Descripción del caso: Femenino de 16 años, en clase funcional II, soplo sistólico grado II foco pulmonar, saturación 96\%. Tomografía de tórax: consolidación peribroncovascular izquierdo. Cateterismo: estenosis pulmonar periférica difusa, defecto de perfusión en vasculatura de lóbulo izquierdo, probable trombosis vs atresia, Pmap $78 \mathrm{mmHg}$. Función sisto-diastólica biventricular conservada. Gammagrama

Figura 15.8.1.
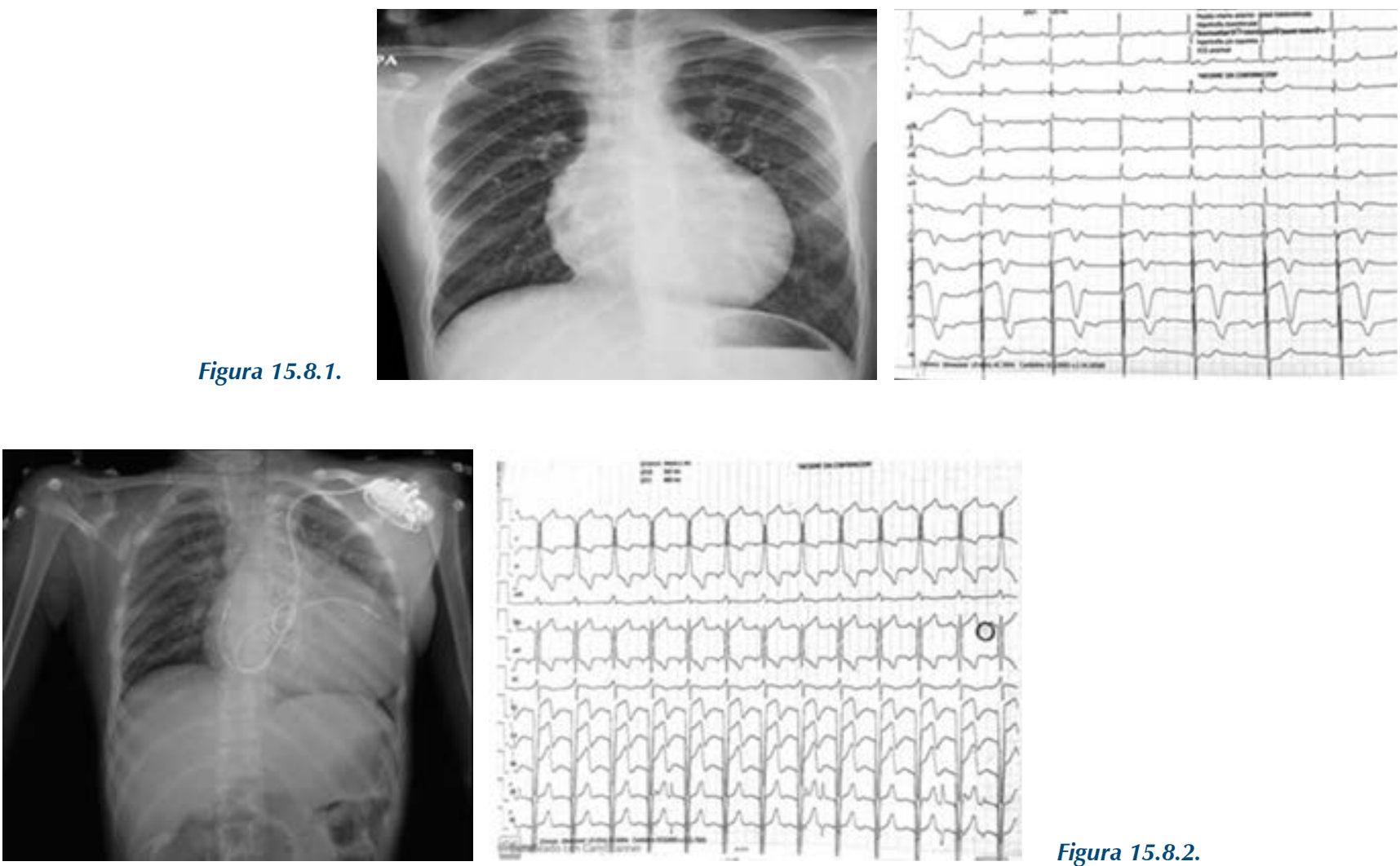

Figura 15.8.2. 
Figura 15.9.1.

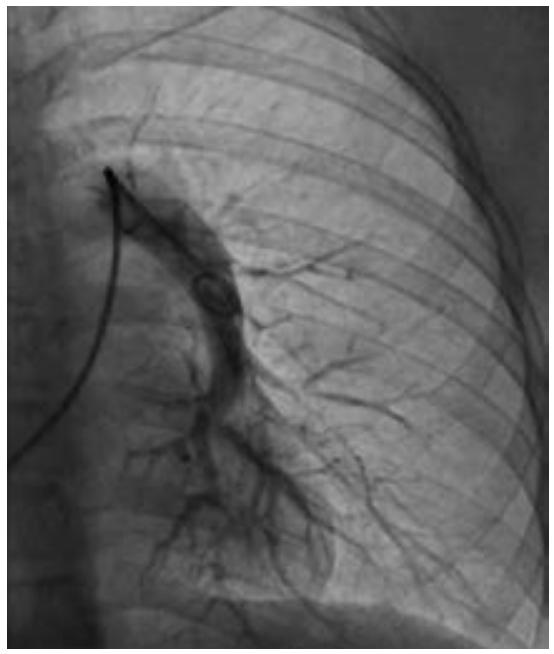

Figura 15.9.2.

pulmonar perfusorio: tromboembolia pulmonar. Metodología: A través del método booleano se realizó búsqueda de términos MeSH «segmental arteries» «pulmonary» [and] «stenosis» en la base de datos con repositorios de evidencia científica arbitraria indizada (PubMed) y no indizadas (Google Scholar). Resultados: Los pacientes con esta enfermedad suelen tener presiones sistémicas del ventrículo derecho secundarias a la obstrucción en las ramas lobular, segmentaria y subsegmental. La colocación de stents de pequeñas arterias segmentarias, particularmente en pacientes con estenosis pulmonar periférica difusa congénita, ha tenido menos éxito. Discusión: En los últimos años la intervención quirúrgica de las arterias pulmonares periféricas se ha asociado con una baja mortalidad y una reducción de la relación de presión VD/Ao casi de $50 \%$. El tratamiento actual de estos pacientes sigue siendo algo controvertido y poco documentado, ya que existen sitios en los cuales no puede abordarse quirúrgicamente.

\subsection{Tromboembolismo pulmonar en endocarditis y endarteritis infecciosa}

García Amaya Carlos Vladimir,* Salazar Lizárraga David,

Germar Arroyo Cristopher, ${ }^{\ddagger}$ López Gallegos Diana, ${ }^{\ddagger}$ García Dávalos Israel ${ }^{\ddagger}$
* Instituto Mexicano del Seguro Social. ${ }^{\ddagger}$ Hospital de Cardiología del Centro Médico Nacional Siglo XXI Cardiología, IMSS.

Tipo de estudio: Reporte de casos clínicos con revisión de fuentes

Introducción: La endocarditis infecciosa es una enfermedad rara, potencialmente devastadora en pacientes pediátricos, principalmente en enfermedad cardiaca congénita preexistente. La causa más frecuente son cocos Gram-positivos. El embolismo pulmonar es poco usual, asociada principalmente a procesos infecciosos. El embolismo séptico pulmonar es el paso de un trombo séptico a la circulación pulmonar, produciendo lesión por toxinas e inflamación. Descripción del caso: Femenino de 15 años con PCA. Se realizó obturación de molares sin profilaxis, posteriormente presentó tos productiva, fiebre, disnea e hipertensión arterial pulmonar. La tomografía mostró trombos pulmonares. ECOTT PCA, endarteritis y endocarditis de válvula pulmonar, HAP. Diagnóstico definitivo de endocarditis y endarteritis infecciosa, trombosis pulmonar. Se manejó tromboendarterectomía, vegetectomía y plastia del tronco de la arteria pulmonar. Cultivo positivo a S. epidermidis. Metodología: Se realizó una búsqueda de fuentes confiables e indexadas
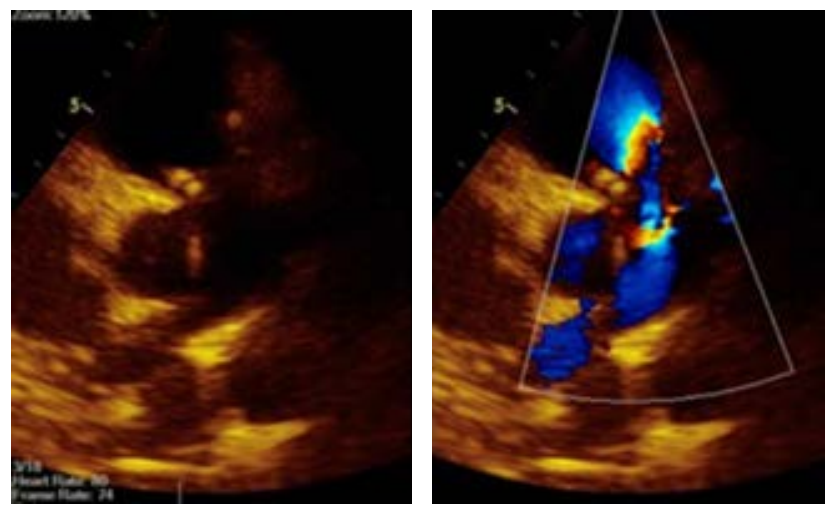

Figura 15.10.1.

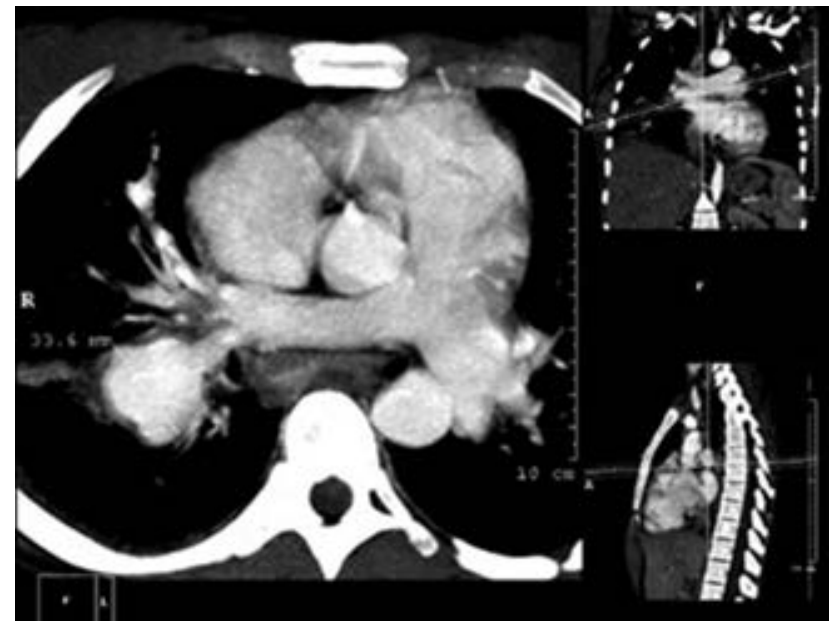

Figura 15.10.2. 
sobre casos de endarteritis y endocarditis infecciosa que generaran trombosis pulmonar en el paciente pediátrico. Se comparó con el caso revisado, tanto la presentación clínica como la frecuencia, el diagnóstico y el manejo. Resultados: En las referencias revisadas, la incidencia de trombosis pulmonar en pediátricos es infrecuente, es un diagnóstico difícil, sobre todo en presentaciones atípicas; la causa más frecuente es infecciosa. Los factores de riesgo fueron cardiopatía congénita previa no tratada y manejo dental sin profilaxis, generó endocarditis, endarteritis y trombosis pulmonar con manejo médico y quirúrgico agresivos. Discusión: La endarteritis y endocarditis infecciosa en el paciente pediátrico es poco frecuente, además de ser un diagnóstico difícil de determinar en presentaciones atípicas. El manejo oportuno es indispensable para evitar secuelas y complicaciones como la trombosis pulmonar. En caso de presentarse requieren manejo inmediato y agresivo como sucedió en nuestro caso.

\subsection{Emergencia hipertensiva como manifestación inicial de coartación aórtica en adolescente}

García Cosio José Carlos, Germán Arroyo Cristopher,

Salazar Lizárraga David, Márquez González Horacio, López Gallegos Diana, Yáñez Gutiérrez Lucelli

Hospital de Cardiología del Centro Médico Nacional Siglo XXI, IMSS.

Tipo de estudio: Reporte de casos clínicos con revisión de fuentes

Introducción: La prevalencia de la coartación aórtica $(\mathrm{CA})$ es de 5-8\% de todos los defectos cardiacos congénitos. La CA genera el desarrollo de hipertensión arterial sistémica (HTA) que afecta al hemicuerpo superior. Clínicamente suele cursar de forma asintomática hasta la segunda o tercera década de vida, a partir de la cual aparecen las complicaciones atribuibles a la HTA. Descripción del caso: Masculino de 11 años, acude por cefalea de severa intensidad, cursa con cifras tensionales encima de percentil 99 y soplo sistólico en área dorsal interescapular izquierda. TAC de cráneo: edema cerebral leve. Ecocardiografía: CA circunscrita. Angio-TAC: CA a nivel descendente. Cateterismo cardiaco: CA con luz mínima de cinco a $20 \mathrm{~mm}$ de la arteria subclavia izquierda. Se realizó angioplastia con stent. Metodología: Se realizó una búsqueda en

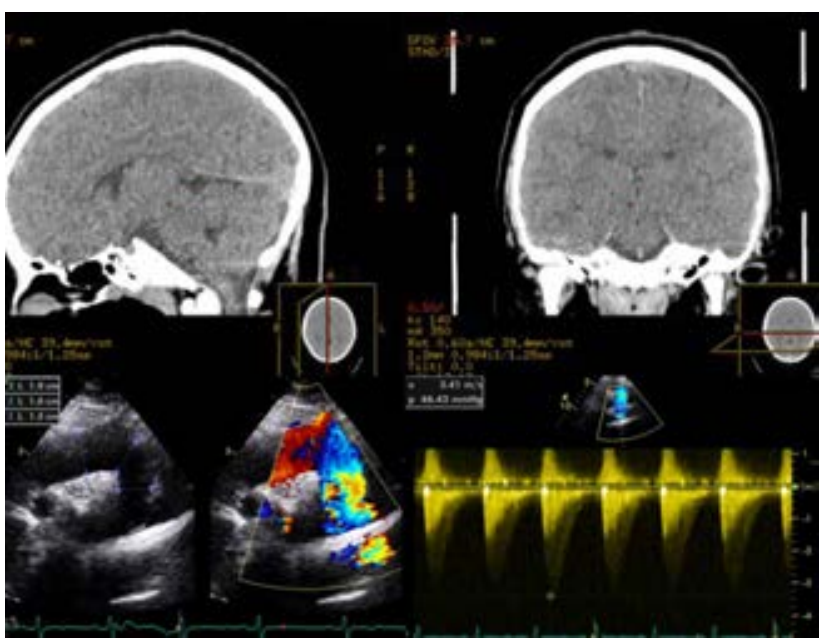

Figura 15.11.1.
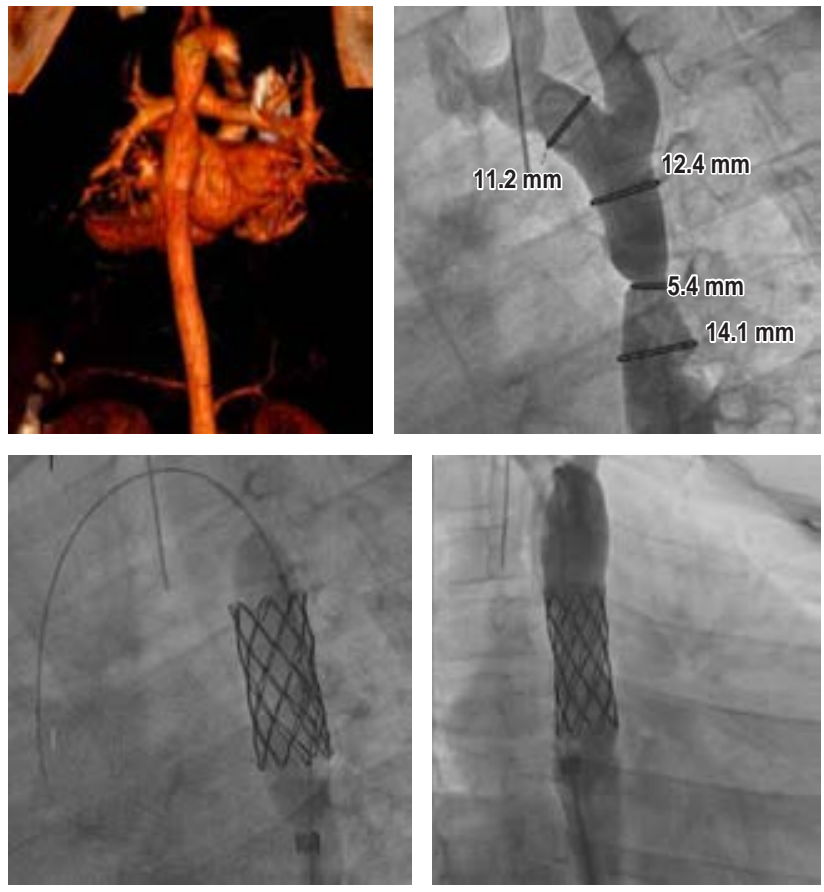

Figura 15.11.2.

PubMed utilizando la palabra clave «hypertensive emergency and aortic coarctation», se limitó la búsqueda a los últimos 20 años, se incluyeron artículos de revisión, ensayos clínicos y metaanálisis. Resultados: La CA determina una obstrucción al flujo sanguíneo, lo que genera el desarrollo de HTA que afecta característicamente al hemicuerpo superior (sectorial), hipertrofia concéntrica del ventrículo izquierdo por sobrecarga sistólica del mismo y el desarrollo de circulación colateral que contribuye a mantener la perfusión del hemicuerpo inferior. El diagnóstico debe sospecharse en aquellos pacientes jóvenes portadores de HTA. Discusión: Este caso muestra la presentación de emergencia hipertensiva con daño a órgano blanco secundaria a una coartación aórtica. Se realiza angioplastia y colocación de stent de forma exitosa con gradiente residual de $0 \mathrm{mmHg}$. Se logra control de cifras tensionales junto con tratamiento médico.

\subsection{Abordaje multidisciplinario en reparación de complicaciones tardías por cirugía de Mustard a 50 años}

Vázquez Salinas Liliana, Yáñez Gutiérrez Lucelli,

Germán Arroyo Cristopher, Salazar Lizárraga David, García Dávalos Israel, Santiago Hernández Jaime, Márquez González Horacio

Hospital de Cardiología del Centro Médico Nacional Siglo XXI, IMSS.

Tipo de estudio: Reporte de casos clínicos con revisión de fuentes

Introducción: La transposición de las grandes arterias (TGA) es la cardiopatía cianótica más grave en el periodo neonatal, con el paso de los años diversas técnicas quirúrgicas se han propuesto, incluidas las reparaciones fisiológicas (Mustard, Senning) y las anatómicas (Jatene), además de intervenciones paliativas quirúrgicas (Blalock- 


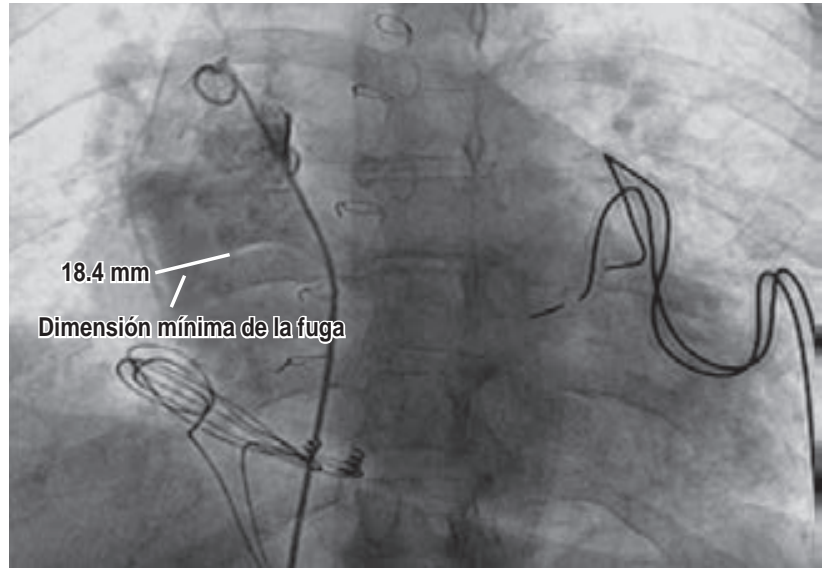

Figura 15.12.1.

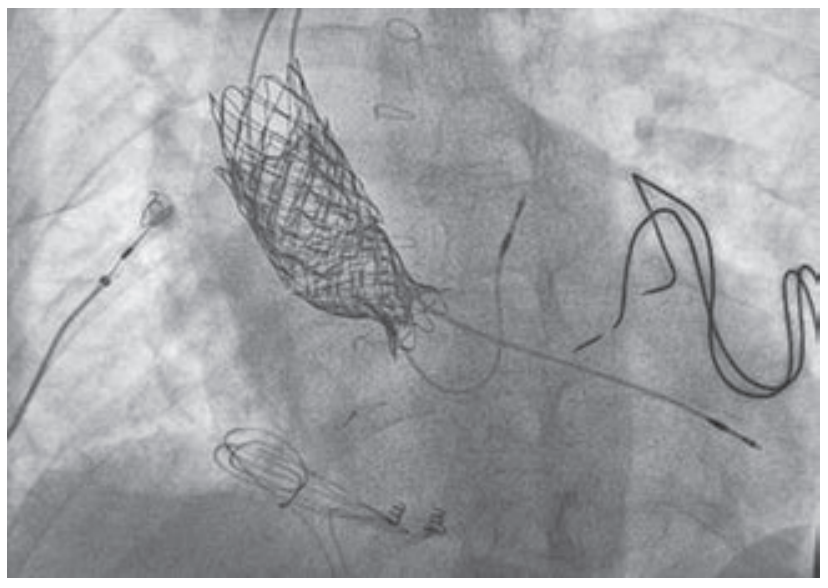

Figura 15.12.2.

Hanlon) o percutáneas (atrioseptostomía de Rashkind). Descripción del caso: Mujer de 52 años, cirugía de Mustard (1970), marcapasos epicárdico, ameritó recambio de generador en ocho ocasiones y nefrectomía por hidronefrosis. CF III y saturación al 70\%. Se documentó la disfunción del túnel superior (venoso sistémico) y disincronía atrioventricular. Abordaje multidisciplinario, colocación de stents recubiertos (CP cubierto 8 y 10 Zig-45 mm), así como cambio de sistema a DDR a través de stents. Metodología: A través del método booleano se realizó búsqueda de términos $\mathrm{MeSH}$ Mustard y transposición de grandes vasos en las bases de datos con repositorios de evidencia científica arbitrada indizada (PubMed) y no indizadas (Google Scholar). Resultados: Las complicaciones de la cirugía de Mustard incluyen disfunción del nódulo sinusal, arritmias, disfunción ventricular derecha sistémica, regurgitación tricuspídea, estenosis pulmonar subvalvular, obstrucciones y fugas del deflector, hipertensión pulmonar y muerte súbita. El pronóstico de vida es aproximado de 30 años. Se encuentra en la categoría de alto riesgo en el embarazo por las complicaciones maternas y fetales. Discusión: Desde 1980 se prefiere la reparación anatómica por las complicaciones tardías, el switch auricular se ha relegado en el mundo pediátrico. Los adultos que sobrevivieron a este tipo de reparaciones requieren manejo multidisciplinario y opciones terapéuticas por cardiología intervencionista y mejores dispositivos de sincronización AV, ya que disminuyen la morbimortalidad comparados con los pacientes tratados convencionalmente con una segunda o tercera intervención quirúrgica.

\subsection{Cierre percutáneo del conducto arterioso persistente en pacientes prematuros y de bajo peso al nacer}

De la O Durán Iris Melina, De Jesús Alvarenga Roberto Teodoro, Bazzoni Ruiz Alberto Esteban, García Reyes Alejandro, Wong Estrello Raúl, Chávez Ortiz Iván, Trejo González Cosme Francisco Unidad Médica de Alta Especialidad No. 71, IMSS Torreón, Coahuila. Tipo de estudio: Reporte de casos clínicos con revisión de fuentes

Introducción: El conducto arterioso persistente (CAP) es una afección común en los neonatos prematuros. El enfoque terapéutico consiste en el tratamiento médico y la ligadura quirúrgica; la tasa de fracaso es de $20-30 \%$ y la mortalidad de 10\%. Desde Porstmann,

Figura 15.13.1.
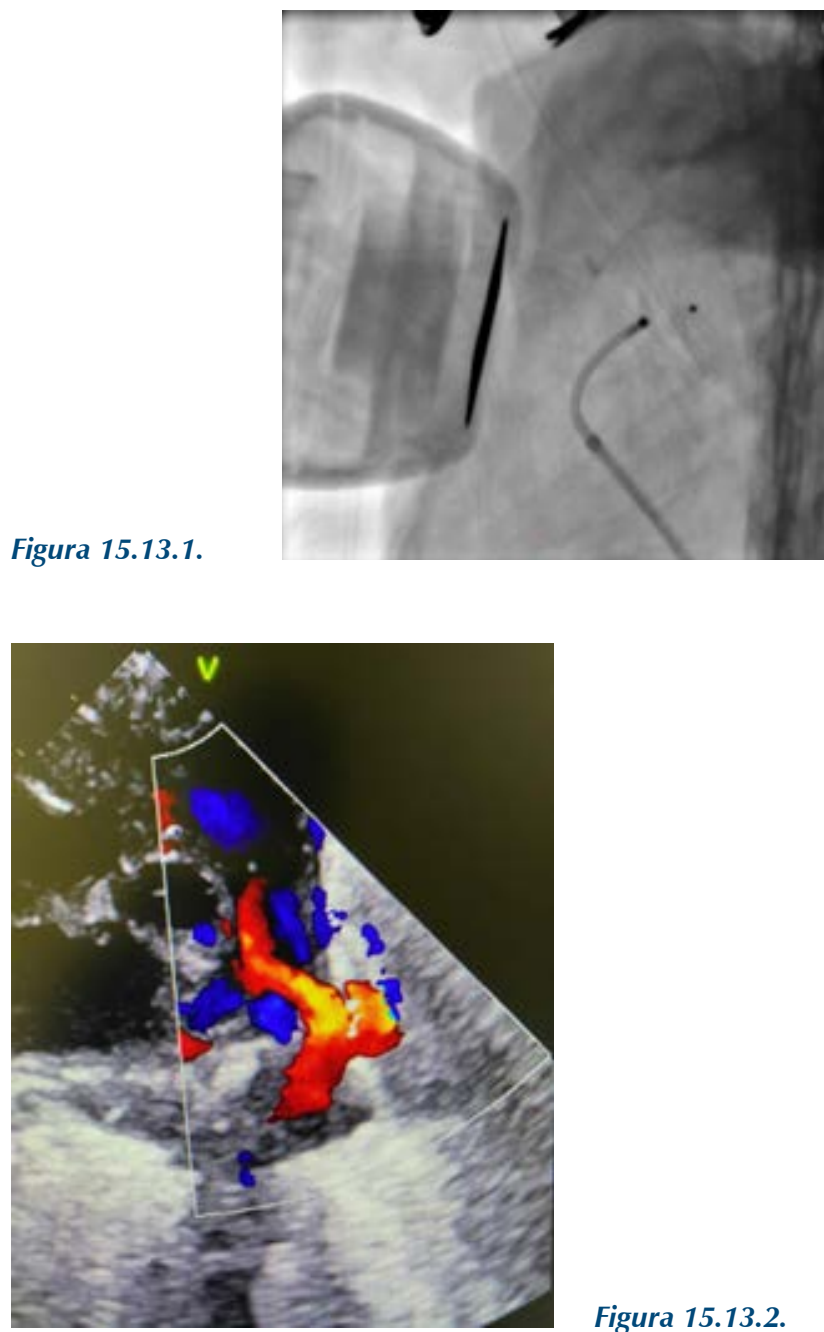

Figura 15.13.2. 
1967, se han logrado mejoras técnicas en la oclusión transcatéter del CAP, aumentando las tasas de éxito, incluso en pacientes prematuros y de bajo peso. Descripción del caso: Presentamos dos neonatos con bajo peso, nacidos mediante cesárea en la semana 28 y 35, respectivamente. Un neonato recibió ibuprofeno, ineficaz. Fueron remitidos a nuestro centro a los 13 y 22 días. Se objetivó la persistencia de ductus (media de $2.75 \mathrm{~mm}$ extremo pulmonar), QP/QS promedio 4:1. Fueron sometidos, por vía anterógrada, a cierre percutáneo de CAP; implante de dispositivos Amplatzer AS. Metodología: Se realizó una búsqueda sistemática de todos los artículos publicados en los últimos 20 años, desde 2000 a 2019, en MEDLINE, PubMed, EMBASE, Google Scholar. La estrategia de búsqueda incluyó los términos de conducto arterioso persistente, oclusión transcatéter, prematuros, peso menor 1500 g. Se realizó búsqueda en las referencias de los artículos incluidos para identificar publicaciones adicionales. Resultados: El riesgo de muerte en neonatos con CAP es más alto que en prematuros con ductus cerrado; por lo tanto, se requiere la oclusión del ductus. El advenimiento de dispositivos de cierre percutáneo, las diferentes técnicas de acceso, el uso combinado de ecocardiograma y fluoroscopia hace factible el cierre mediante cateterismo del ductus en pacientes prematuros y de muy bajo peso. Discusión: Se describen dos neonatos prematuros que se sometieron a cierre exitoso del conducto arterioso transcatéter, no presentaron complicaciones; en el examen ecocardiográfico posterior al procedimiento, sin flujo residual. La oclusión transcatéter de conducto arterioso persistente hemodinámicamente significativo permitió una intervención temprana, disminuyó los días de estancia y demostró ser un procedimiento seguro y eficaz.

\subsection{Fístula de arteria coronaria derecha asociada con aneurisma del seno de Valsalva derecho}

García Rosales Horacio,

Germán Arroyo Cristopher, García Dávalos Israel, Salazar Lizárraga David, Márquez González Horacio, Yáñez Gutiérrez Lucelli

Instituto Mexicano del Seguro Social.

Tipo de estudio: Reporte de casos clínicos con revisión de fuentes

Introducción: Las fístulas coronarias son anomalías poco frecuentes caracterizadas por una conexión anormal entre las arterias coronarias y las estructuras vasculares o cavidades cardiacas. La ligadura electiva en la infancia se recomienda debido al riesgo de complicaciones tardías. Desde el primer cierre transcatéter exitoso hace 37 años, existen pocas descripciones de resultados a largo plazo. Descripción del caso: Masculino de 16 años. Soplo al nacimiento. Diagnóstico: fístula coronaria derecha-ventrículo derecho; oclusión con coil; se documentó flujo de fístula coronaria con circulación colateral septum-inferior y ventrículo derecho, dilatación aneurismática del seno de Valsalva. Angiografía: recanalización distal por la arteria del cono, aneurisma del seno de Valsalva derecho no roto $(43 \mathrm{~mm})$. Se realizó cierre percutáneo (microcoil, exitoso) y vigilancia del aneurisma. Metodología: A través del método booleano se realizó búsqueda de términos $\mathrm{MeSH}$ «Complications» [and] «Coronary artery fistulas» en las bases de datos con repositorios de evidencia científica arbitraria indizada (PubMed) y no indizadas (Google Scholar). Resultados: El cierre percutáneo con colocación de coils es una modalidad de tratamiento segura y eficaz. Durante el seguimiento se ha reportado caso de recanalización de la fístula, lo que deriva en la importancia del seguimiento después del cierre, incluso en asintomáticos. Discusión: Se ha reportado formación
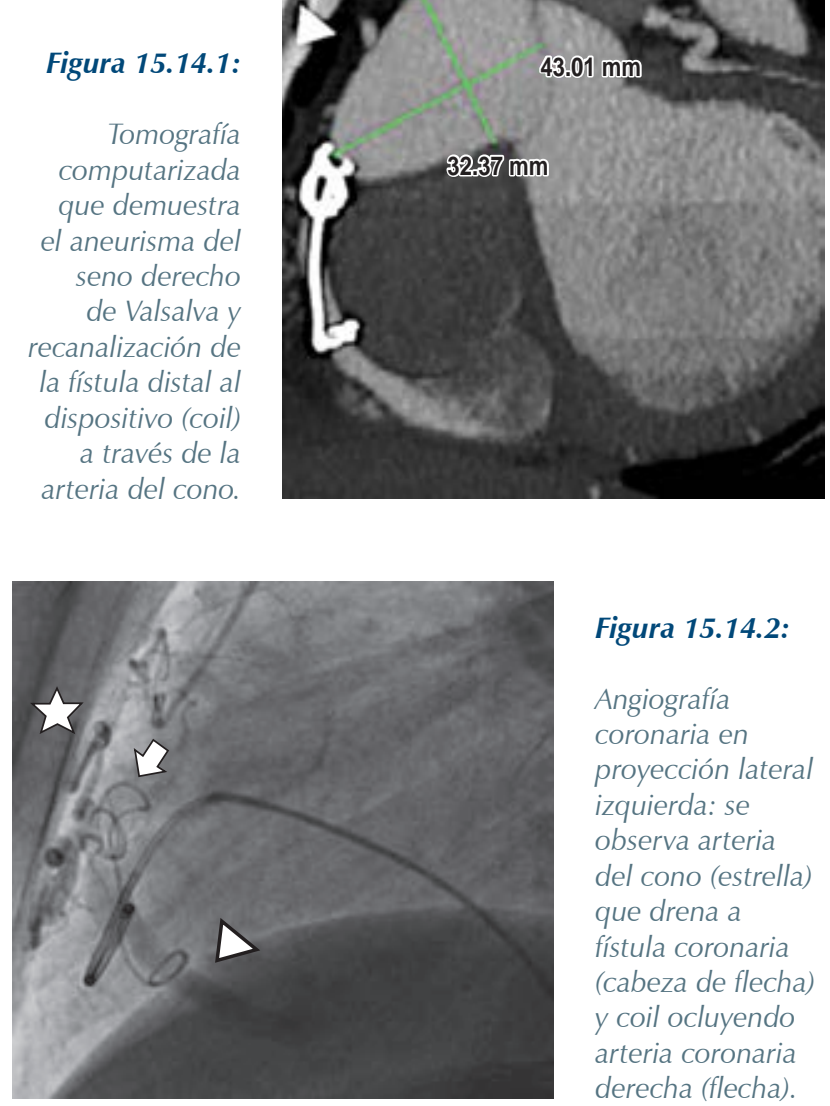

Figura 15.14.2:

Angiografía coronaria en proyección lateral izquierda: se observa arteria del cono (estrella) que drena a fístula coronaria (cabeza de flecha) y coil ocluyendo arteria coronaria derecha (flecha).

de aneurismas en 9\% de los pacientes no tratados; sin embargo, no existen publicaciones respecto a la asociación de recanalización de la fístula con aneurisma del seno de Valsalva, ni de complicaciones a largo plazo de estos casos.

\subsection{Cirugía de triple implante valvular en adolescente con corrección de tetralogía de Fallot}

Miranda Salgado María Guadalupe, Guerrero Hernández Alejandra, Vázquez Salinas Liliana, Germán Arroyo Cristopher,

Salazar Lizárraga David, García Dávalos Israel

Hospital de Cardiología del Centro Médico Nacional Siglo XXI, IMSS.

Tipo de estudio: Reporte de casos clínicos con revisión de fuentes

Introducción: La insuficiencia de la válvula pulmonar en el paciente postoperado de corrección total en la tetralogía de Fallot debe tener seguimiento estrecho, debido a que la insuficiencia pulmonar conduce a dilatación ventricular derecha y disfunción biventricular, que además se asocia con la presencia de arritmias severas y muerte súbita. Descripción del caso: Femenino de 13 años. Antecedente de tetralogía de Fallot reparado a los cuatro meses de edad, en clase funcional III; miocardiopatía dilatada con insuficiencia mitral, tricuspídea y pulmonar severas, con disfunción sisto-diastólica biventricular en seguimiento ecocardiográfico. Se somete a recambio trivalvular mecánico de las válvulas: pulmonar, tricúspide y mitral. Metodología: A través del método booleano se realizó 

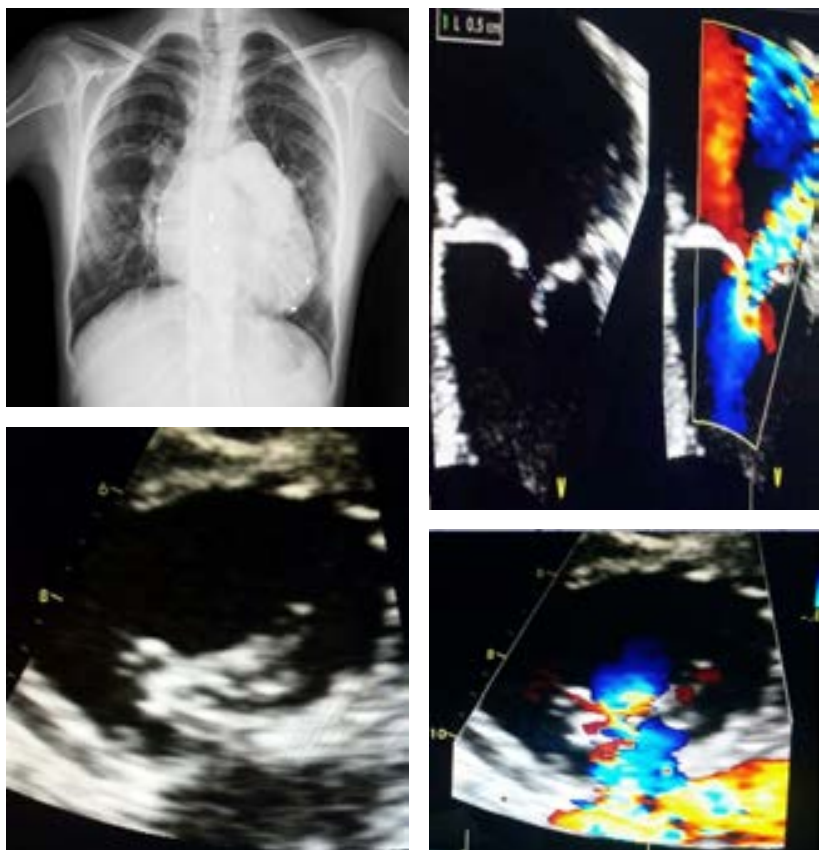

Figura 15.15.1.

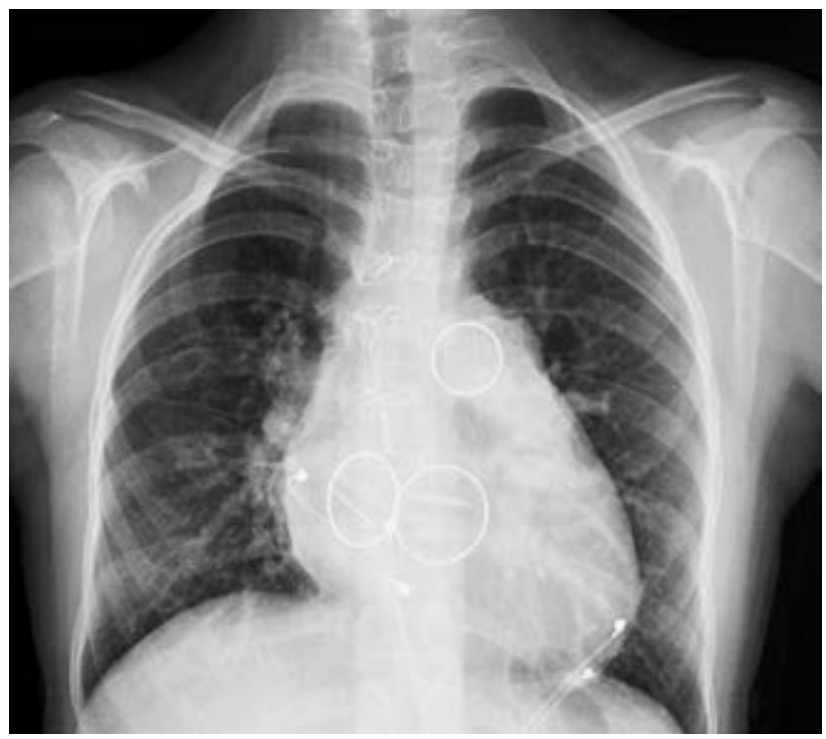

Figura 15.15.2.

búsqueda de términos $\mathrm{MeSH}$ sobres casos de recambios valvulares mecánicos en paciente pediátrico postoperado de reparación total de tetralogía de Fallot, en las bases de datos con repositorios de evidencia científica arbitraria indizada (PubMed) y no indizadas (Google Scholar). Se comparó con el caso revisado la presentación clínica, frecuencia, diagnóstico y manejo. Resultados: En las referencias revisadas la frecuencia de recambio de implante valvular mecánico múltiple es infrecuente, siendo el manejo y evolución de estos pacientes muy insidioso, así como el seguimiento para el control de la anticoagulación. Los factores de riesgo de los pacientes fueron disfunción sisto-diastólica biventricular, así como presencia de arritmias y la falta de apego al tratamiento con anticoagulantes. Discusión: Existe poca evidencia sobre la experiencia en el manejo del paciente pediátrico con implantes valvulares mecánicos y el apego a la anticoagulación oral; sin embargo, el poder tratar a este tipo de pacientes nos permite obtener esa experiencia para poder ofrecer un adecuado seguimiento y control del paciente pediátrico con múltiples implantes valvulares mecánicos.

\subsection{Transposición congénitamente corregida de grandes vasos en paciente con angina inestable}

Macossay Santiago Sigifredo, Salazar Lizárraga David,

García Dávalos Israel, Germán Arroyo Cristopher, Ramírez Sánchez Patricia, Mercado Rojas Gerardo, Pacheco López Alejandro

UMAE Hospital de Cardiología del Centro Médico Nacional Siglo XXI

Tipo de estudio: Reporte de casos clínicos con revisión de fuentes

Introducción: La transposición congénitamente corregida de grandes vasos (TCCGV) es una condición muy rara que se caracteriza por discordancia atrioventricular y ventrículo arterial. Representa menos de $1 \%$ de todas las anomalías congénitas. Existen pocos reportes previos de pacientes con TCCGV y angina inestable. Descripción del caso: Hombre de 58 años, antecedentes: hipertensión arterial sistémica y tabaquismo. Acude por dolor torácico típico. El electrocardiograma muestra bloqueo de rama izquierda, sin elevación del ST. Troponina T normal. Ecocardiograma: concordancia auriculoventricular, discordancia ventriculoarterial tipo transposición de grandes vasos corregida. FEVI 50\%, hipocinesia anterior tres segmentos. Angiotomografía: lesiones coronarias no
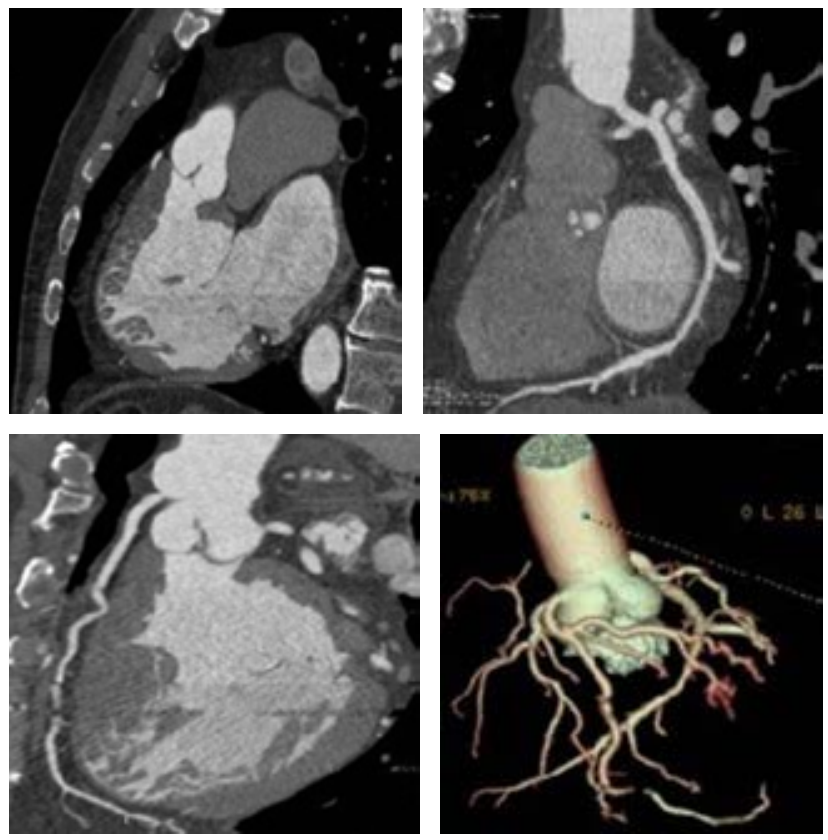

Figura 15.16.1. 

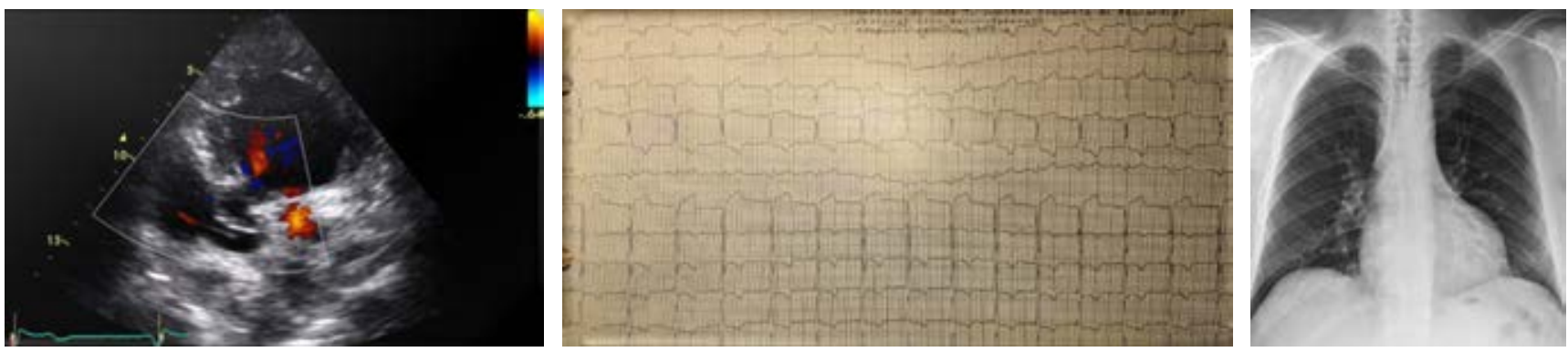

Figura 15.16.2.

significativas, calcio coronario 81 unidades Agatston. Metodología: A través del método booleano se realizó búsqueda de términos $\mathrm{MeSH}$ «congenitally corrected transposition of great arteries» [and] «unstable angina» en las bases de datos con repositorios de evidencia científica arbitrada indizada (PubMed) y no indizadas (Google Scholar). Resultados: En la TCCGV la coronaria derecha irriga al ventrículo derecho, que se encarga de la circulación sistémica y se dilata o presenta hipertrofia, con esto, el aporte de sangre resulta insuficiente con un desequilibrio oferta-demanda de oxígeno, causando isquemia representada por dolor torácico. Discusión: Este caso muestra a un paciente con dolor torácico en contexto de cardiopatía congénita compleja. Tradicionalmente, la etiología más común de los síntomas es la ateromatosis. Es estratificado como riesgo intermedio, se realiza angiotomografía coronaria, la cual descarta dicha causa. La angiotomografía de arterias coronarias es un método no invasivo muy valioso para visualizar de manera precisa la anatomía coronaria.

\subsection{Obstrucción del túnel superior e inferior en paciente postoperado de Senning-Mustard reparado con D}

Mercado Rojas Gerardo,Almeida GutiérrezEduardo, OsorioAgosto JorgeRafael, Gómez González Daniel Alejandro, Ramírez Sánchez Patricia,

Pacheco López Alejandro, Macossay Santiago Sigifredo UMAE Hospital de Cardiología del Centro Médico Nacional Siglo XXI. Tipo de estudio: Reporte de casos clínicos con revisión de fuentes

Introducción: La cirugía de Mustard consiste en la escisión del septo interatrial y creación de uno nuevo mediante parche de pericardio para derivar el retorno venoso sistémico a través de la válvula mitral al ventrículo izquierdo y a la arteria pulmonar, y el retorno venoso pulmonar a través de la válvula tricúspide al ventrículo derecho y a la circulación sistémica. Descripción del caso: Mujer de 21 años diagnosticada al nacer con transposición de los grandes vasos, se realiza switch atrial tipo Mustard a los cuatro meses. Refiere hace cuatro años deterioro de su clase funcional, cateterismo cardiaco con obstrucción del túnel superior $(17 \mathrm{mmHg}$ ) y del túnel inferior (13 mmHg). Metodología: A través del método booleano se realizó búsqueda de términos MeSH «Transposition of the great arteries after mustard repair» [and] «Mustard baffle obstruction and leak» en la base de datos con repositorios de evidencia científica arbitrada indizada (PubMed) y no indizadas (Google Scholar). Resultados: Las complicaciones asociadas a la cirugía se manifiestan a los 2030 años desde la corrección, en este caso la obstrucción del baffle de Mustard puede encontrarse en $60 \%$ de estos pacientes como
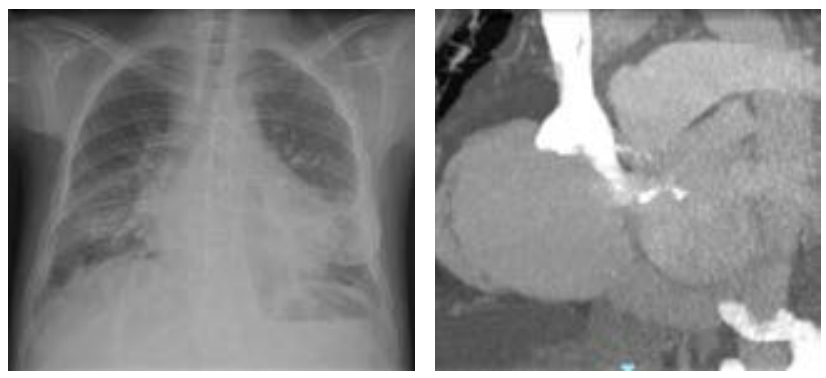

Figura 15.17.1.
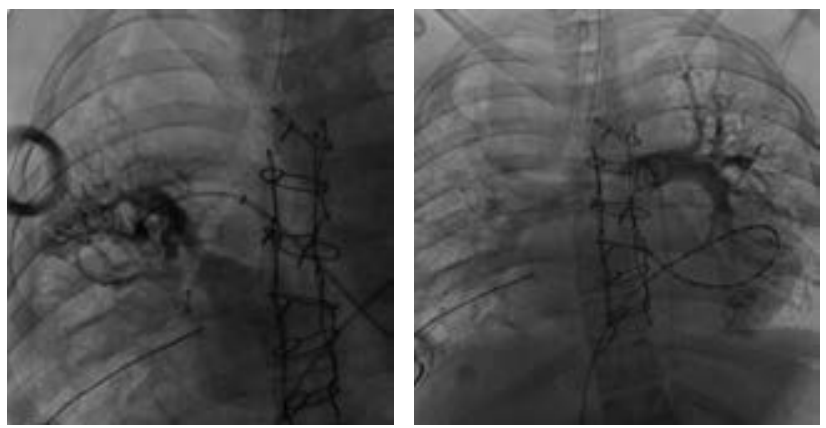

Figura 15.17.2.

complicación y es algo que se debe sospechar en estos sujetos. Discusión: Esta técnica fue descrita en 1964 por W. Mustard, denominada corrección oximétrica. En la actualidad, se emplea como parte de la corrección en el doble switch en la inversión ventricular. Se presenta el caso de una obstrucción de ambos túneles con desmantelamiento y reparación de Mustard realizada de manera exitosa.

\subsection{Supervivencia a las fallas del sistema de Fontán en pacientes con corazón univentricular}

Márquez González Horacio, Miranda Salgado Guadalupe,

Germán Arroyo Cristopher, García Dávalos Israel, Salazar Lizárraga David, Yáñez Gutiérrez Lucelli

Hospital de Cardiología del Centro Médico Nacional Siglo XXI.

Tipo de estudio: Estudio pronóstico 
Tabla 15.18.1: Epidemiología de las fallas del sistema de Fontán.

\begin{tabular}{lcccc} 
Tipo de falla & $\mathbf{n}$ & $\begin{array}{c}\text { Incidencia acumulada } \\
\text { (caso } \times \mathbf{1 0 0} \text { pacientes) }\end{array}$ & $\begin{array}{c}\text { Densidad de incidencia } \\
\text { (casos por cada 10 años) }\end{array}$ & $\begin{array}{c}\text { HR (IC 95\%) } \\
\text { de muerte }\end{array}$ \\
\hline Cianosis & 12 & 21 & 13 & $3.5(1.5-15)$ \\
Falla del ventrículo único & 5 & 9 & 10 & $1.9(1.2-5)$ \\
Enteropatía perdedora de proteínas & 3 & 5 & 8 & $2.3(2.3-7)$ \\
Bronquitis plástica & 2 & 4 & 2 & $1.2(0.9-4)$ \\
\hline
\end{tabular}

Incidencia acumulada = casos/total de expuestos. Densidad de incidencia = casos/tiempo persona (años)

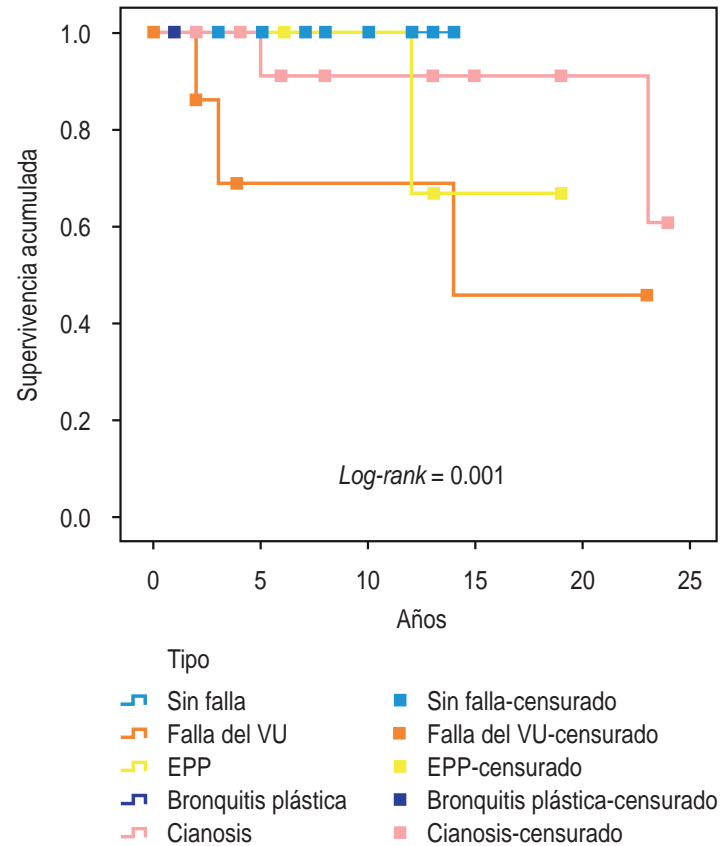

Figura 15.18.1: Probabilidad de estar libre de muerte según el tipo de falla del sistema de Fontán.

Introducción: El sistema de Fontán (SF) es el procedimiento quirúrgico de elección para reparar las cardiopatías congénitas univentriculares (CCU), consiste en la conexión de ambas venas cavas a la arteria pulmonar. En 2019 se actualizaron los criterios de falla del SF, los principales son: disfunción del ventrículo único (DVU), cianosis, enteropatía perdedora de proteínas (EPP) y bronquitis plástica (BP). Objetivo: Determinar la incidencia acumulada (IA), densidad de incidencia (DI), razón de riesgos (HR) y supervivencia de las fallas del SF en una cohorte de pacientes con CCU. Metodología: Estudio de cohorte de pacientes reparados con SF de la clínica del corazón univentricular de un centro de tercer nivel. El tiempo cero se consideró al egreso exitoso de la cirugía, las variables de desenlace fueron: DVU (fracción de expulsión menor de 50\%), cianosis (saturación periférica $<85 \%$ ), EPP (hipoalbuminemia, alteración en pruebas de función hepática) y BP (broncoscopia). Resultados: Análisis estadístico: medidas de tendencia central y dispersión. Cálculo de IA, DI y supervivencia. Resultados: La cohorte se integró por 56 pacientes con una mediana de edad de 15 (5-39) años y seguimiento de cinco (1-25) años; la atresia tricuspí- dea representó 30\% de las CCU; 29 (51\%) pacientes no presentaron criterio de falla. La mortalidad se presentó en seis (10\%). Las medidas epidemiológicas se presentan en la Tabla 15.18.1 y la función de supervivencia en la Figura 15.18.1. Discusión y conclusiones: En esta cohorte la DVU fue la causa más frecuente y de mayor riesgo de mortalidad, es preciso ajustar los manejos terapéuticos a esta causa.

\subsection{Factores asociados con disfunción de prótesis biológicas en pacientes con cardiopatías congénitas (CC)}

Márquez González Horacio, Vázquez Salinas Liliana,

Germán Arroyo Cristopher, García Dávalos Israel,

Salazar Lizárraga David, Yáñez Gutiérrez Lucelli

UMAE Hospital de Cardiología del Centro Médico Nacional Siglo XXI.

Tipo de estudio: Estudio pronóstico

Introducción: Las CC tienen con frecuencia alteraciones embriológicas que condicionan lesiones en los aparatos valvulares y que requieren

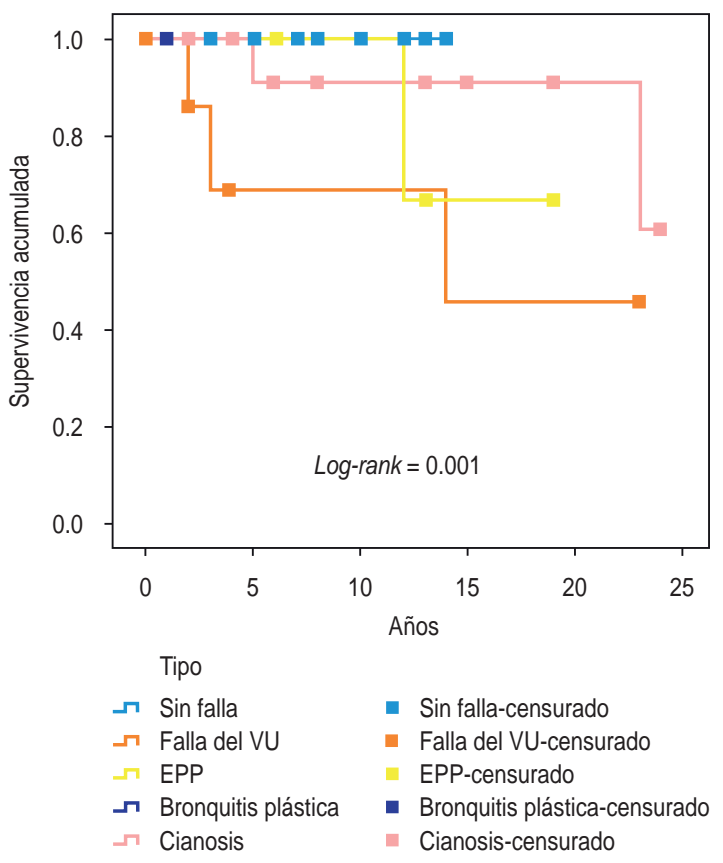

Figura 15.19.1: Probabilidad de estar libre de muerte según el tipo de falla del sistema de Fontán. 


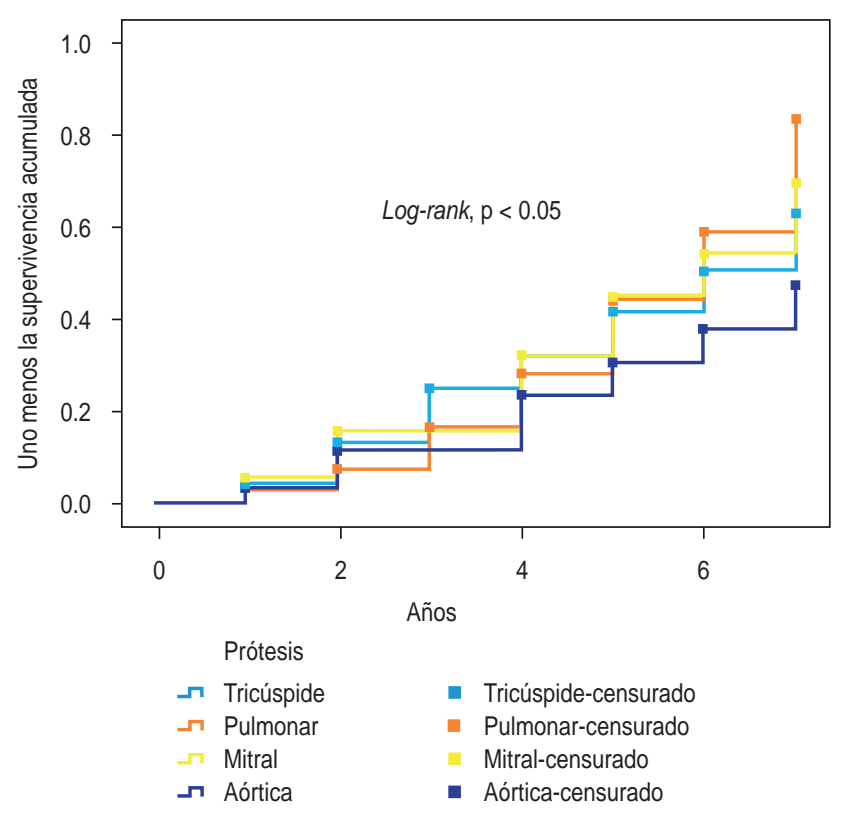

Figura 15.19.2: Probabilidad de presentar falla según la posición de la prótesis.

para su reparación la colocación de prótesis habitualmente biológicas con el fin de permitir que durante la infancia los factores del crecimiento pondoestatural permitan que se coloque una prótesis definitiva. La vida media de las prótesis son de cinco años. Objetivo: Determinar la supervivencia y los factores asociados a la disfunción de prótesis biológica en pacientes reparados de CC. Metodología: Cohorte de pacientes con CC reparados con colocación de valvular biológico en las válvulas atrioventriculares o ventriculoarteriales. El tiempo cero de la cohorte se consideró al egreso exitoso; la variable de desenlace fue la disfunción de la prótesis (ecocardiográficos y clínica). Variables de interés: edad, posición de la prótesis, CC de base, marca de la prótesis, uso de anticoagulante o antiagregante, antropometría. Resultados: Análisis estadístico: cálculo de riesgos por HR ajustado por modelo de Cox a un confusor, curva de supervivencia; 253 pacientes con mediana de edad de 11 (6-36) años, 132 (52\%) fueron mujeres. Las CC más frecuentes fueron tetralogía de Fallot (57\%), canal atrioventricular (21\%) y aorta bivalva (16\%). La supervivencia se muestra en las Figuras 15.19.1 y 15.19.2. La prótesis con mayor probabilidad de disfunción fue la pulmonar (80\%) a seis años. La mediana de duración de las prótesis fue menor a 10 años. Discusión y conclusiones: Las CC cianóticas con prótesis pulmonar tienen una menor supervivencia, está relacionada la velocidad de crecimiento y el uso de antiagregantes.

\section{Trabajo ganador del premio Dr. Carlos Pérez Treviño Primer Lugar}

\subsection{Escalas para pronosticar la recuperación de la falla ventricular derecha en cardiopatías congénitas}

Márquez González Horacio,* Yáñez Gutiérrez Lucelli,* Garduño Espinosa Juan, ${ }^{\ddagger}$ Vargas Becerra Mario Humberto, ${ }^{\S}$ López Gallegos Diana,* Riera Kinkel Carlos*
* UMAE Hospital de Cardiología del Centro Médico Nacional Siglo XXI. ${ }^{\ddagger}$ Hospital Infantil de México. ${ }^{\S}$ Instituto Nacional de Enfermedades Respiratorias (INER).

Tipo de estudio: Estudio pronóstico

Introducción: La supervivencia en las cardiopatías congénitas (CC) depende de la reparación quirúrgica, algunas al momento de repararse presentan datos de falla del ventrículo derecho (FVD) y son múltiples las variables que intervienen en la recuperación. De persistir, la supervivencia se ve comprometida. Los pacientes con CC requieren múltiples intervenciones, por lo que se requiere sustento para tomar las decisiones. Objetivo: Construir y validar escalas pronósticas para determinar la recuperación de la FVD en seis CC (PCA, CIA, CIV, anomalía de Ebstein, TF, AP + CIV) a dos años de la cirugía de reparación total. Metodología: Dos cohortes (creación y validación) de pacientes con CC que fueron reparados con FVD (parámetros ecocardiográficos) al momento de la cirugía. El tiempo cero fue al momento de egreso del quirófano. Variables de interés: edad, procedimiento, mecanismo, variables ecocardiográficas, defectos residuales, arritmias, bloqueo postquirúrgico. Seguimiento por dos años; el desenlace fue la recuperación de la FVD por ecocardiograma. Resultados: Análisis por fases: creación (bivariado), integración (regresión logística), validación (AUC) y supervivencia. La cohorte de creación se conformó de 720 y la de validación de 138. La persistencia de la FVD a dos años se presentó en la cohorte de creación en $23.5 \%$ de los pacientes vs $38.4 \%$ en la cohorte de validación $(p=0.05)$. Se calculó la probabilidad individual (basado en fórmula de regresión logística) según la cardiopatía, mecanismo de falla y del modelo global (calculadora para descargar: https:// feerrivera.github.io/cardio/). La enfermedad con peor pronóstico fue la anomalía de Ebstein. Discusión y conclusiones: Se obtuvieron modelos matemáticos para predecir la probabilidad de continuar con la FVD sistólica o diastólica, respectivamente, validados en una cohorte prospectiva. El mecanismo sistólico tiene peor pronóstico.

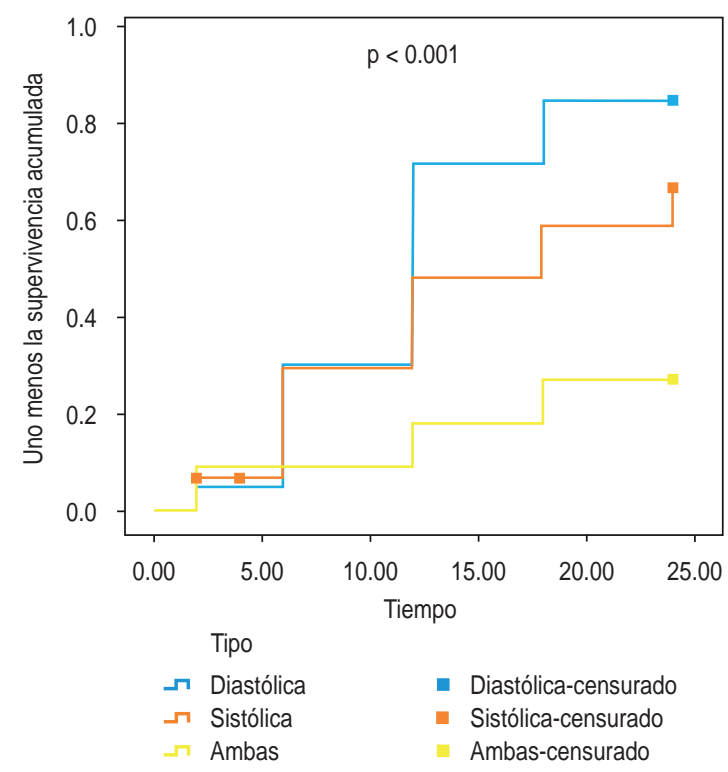

Figura 15.20.1: Probabilidad de recuperar la función de VD. 
Tabla 15.20.1: Modelos pronósticos de la recuperación del FVD en pacientes reparados de CC.

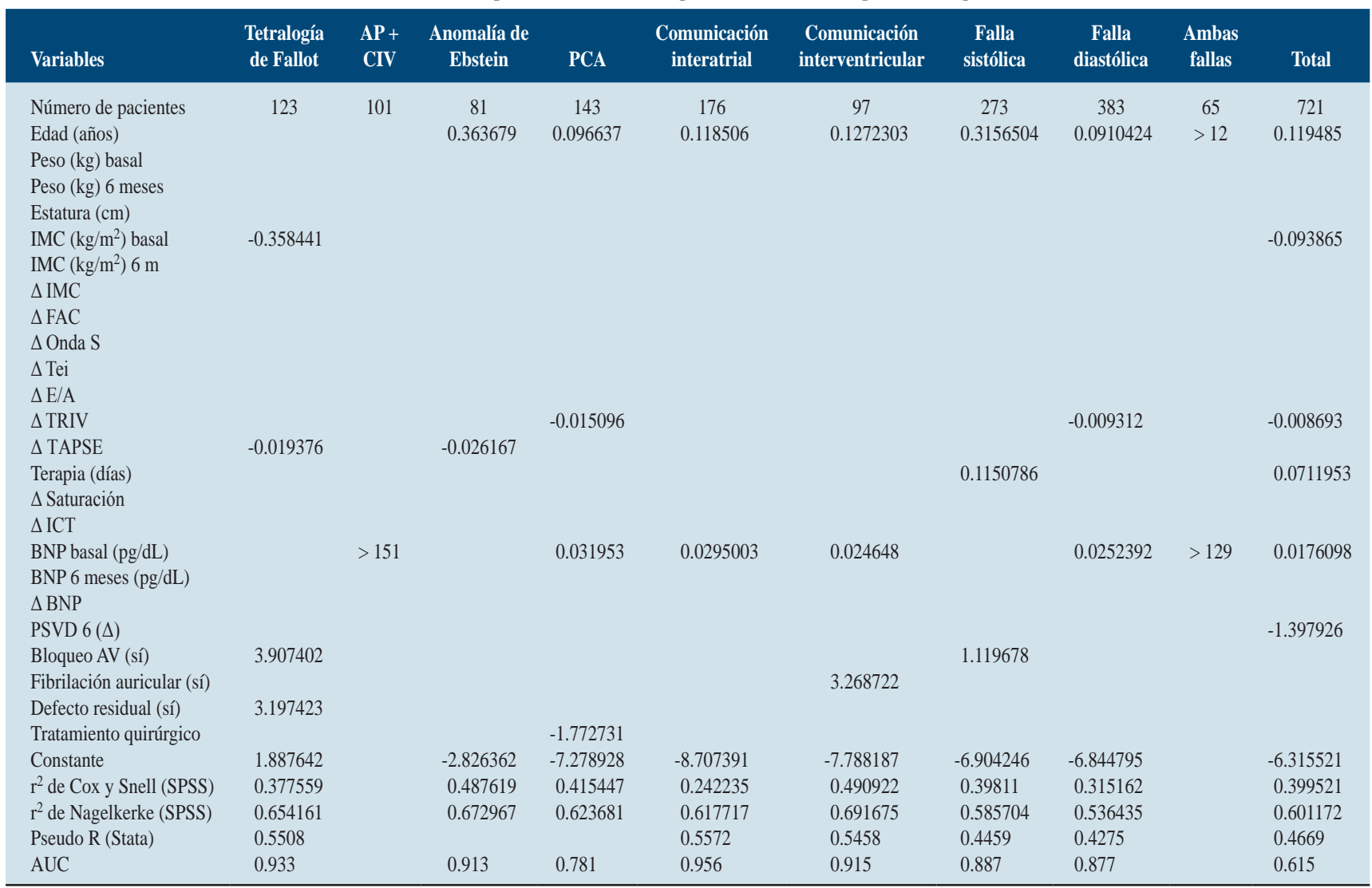

En cada columna, los datos corresponden a los coeficientes de regresión $(\beta)$ de cada una de las variables que quedaron en el modelo final de regresión logística múltiple, con fórmula $\mathrm{p}=\mathrm{e}^{\mathrm{X} 1}\left(1-\mathrm{e}^{\mathrm{X}}\right)$, donde $\mathrm{X}=$ constante $+\beta_{1} \mathrm{X}_{1}+\beta_{2} \mathrm{X}_{2}+\ldots \beta_{\mathrm{n}} \mathrm{X}_{\mathrm{n}}$. Adicionalmente se muestra el coeficiente $\mathrm{r}^{2}$ de Nagelkerke. AUC corresponde al cálculo individual de los sujetos según el modelo pronóstico expresado en porcentaje de probabilidad de presentar el desenlace.

Tabla 15.20.2.

\begin{tabular}{lcccc} 
& \multicolumn{2}{c}{ Cohorte de creación } & \multicolumn{2}{c}{ Cohorte de validación } \\
\cline { 2 - 5 } Enfermedad & AUC & IC-95\% & AUC & IC-95\% \\
\hline PCA & 0.78 & $0.68-0.82$ & 0.56 & $0.54-0.6$ \\
CIA & 0.95 & $0.86-0.97$ & 0.72 & $0.68-0.75$ \\
CIV & 0.91 & $0.89-0.93$ & 0.75 & $0.72-0.77$ \\
TF & 0.93 & $0.89-0.94$ & 0.74 & $0.73-0.78$ \\
AE & 0.91 & $0.89-0.95$ & 0.91 & $0.89-0.91$ \\
Mecanismo sistólico & 0.86 & $0.83-0.87$ & 0.86 & $0.83-0.87$ \\
Mecanismo diastólico & 0.87 & $0.86-0.90$ & 0.57 & $0.55-0.59$ \\
Global & 0.61 & $0.59-0.63$ & 0.75 & $0.73-0.75$ \\
\hline
\end{tabular}

PCA = persistencia del conducto arterioso, CIA = comunicación interatrial, $\mathrm{CIV}=$ comunicación interventricular, $\mathrm{TF}$ = tetralogía de Fallot, $\mathrm{AE}$ = angioedema.

\subsection{Coartación de aorta en el paciente adulto. Reporte de caso}

Miranda Salgado María Guadalupe, Guerrero Hernández Alejandra, Salinas Vázquez Liliana, García Dávalos Israel, Salazar Lizárraga David, Germán Arroyo Cristopher
Hospital de Cardiología del Centro Médico Nacional Siglo XXI, IMSS. Tipo de estudio: Reporte de casos clínicos con revisión de fuentes

Introducción: La coartación de aorta es una patología frecuente que disminuye la expectativa de vida, tiene una prevalencia de 0.2 por 1,000 nacidos vivos, con predominio en el género masculino en México. Descripción del caso: Masculino de 47 años de edad con los diagnósticos de hipertensión arterial sistémica (HAS) de 30 años de evolución, coartación yuxtaductal aórtica, migración de stent aórtico (2002), por cateterismo en 2017 se evidenció la migración de stent aórtico. En julio de 2019 presentó deterioro de la clase funcional II y palpitaciones, se realizó nuevo cateterismo con reporte de un aneurisma anterior. Metodología: A través del método booleano se realizó búsqueda de términos MeSH sobre casos de coartación aórtica en el adulto, en las bases de datos con repositorios de evidencia científica arbitraria indizada (PubMed) y no indizadas (Google Scholar). Se comparó con el caso revisado la presentación clínica, frecuencia, diagnóstico y manejo. Resultados: La presencia de coartación aórtica en el paciente adulto es infrecuente, debido a que su diagnóstico es temprano; sin embargo, en el adulto se trata por intervencionismo para evitar sobreagregar más complicaciones de las que ya cuenta. Discusión: La coartación de aorta representa una entidad patológica que precisa de seguimiento 


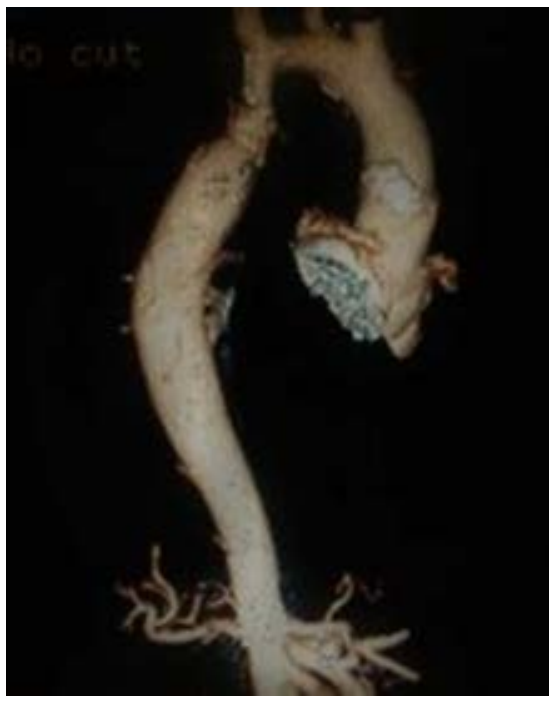

Figura 15.21.1.

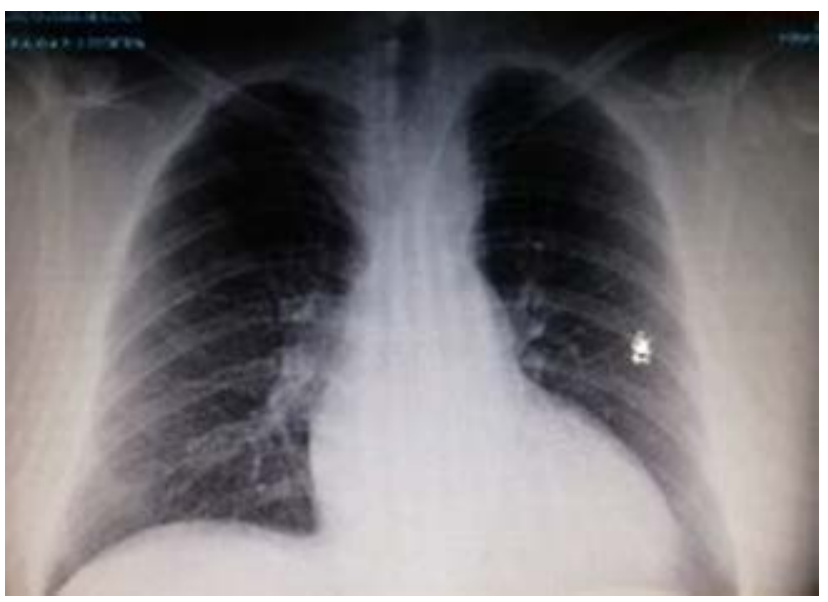

Figura 15.21.2.

permanente y de la cual la presencia de HAS puede ser el signo aislado principal para sospechar en la entidad nosológica.

\subsection{Supervivencia en una clínica de transición de cardiopatías congénitas}

Márquez González Horacio, López Gallegos Diana,

Germán Arroyo Cristopher, Salazar Lizárraga David, García Dávalos Israel, Yáñez Gutiérrez Lucelli

UMAE Hospital de Cardiología del Centro Médico Nacional Siglo XXI.

Tipo de estudio: Estudio pronóstico

Introducción: Las cardiopatías congénitas (CC) tienen en la actualidad una supervivencia mayor a $85 \%$ a 40 años. Las clínicas de transición tienen como finalidad garantizar un adecuado seguimiento multidisciplinario de las complicaciones tardías. En México el proceso de atención es tardío e interrumpido cuando se llega a la edad adulta y las causas de las comorbilidades exigen

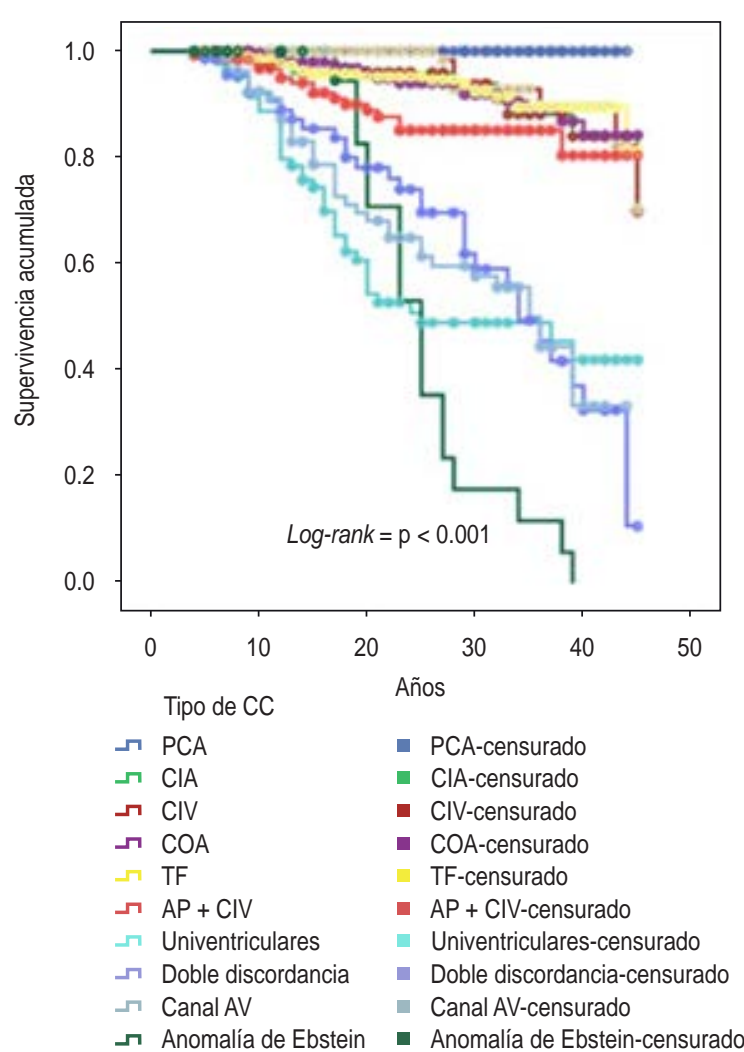

Figura 15.22.1: Supervivencia global de una cohorte de pacientes con CC. $P C A=$ persistencia del conducto arterioso; $C I A=$ comunicación interatrial; $C I V=$ comunicación interventricular; $\operatorname{Co} A=$ coartación aórtica; $T F=$ tetralogía de Fallot; $A P+C I V=$ atresia pulmonar con comunicación interventricular; canal $A V=$ canal atrioventricular.

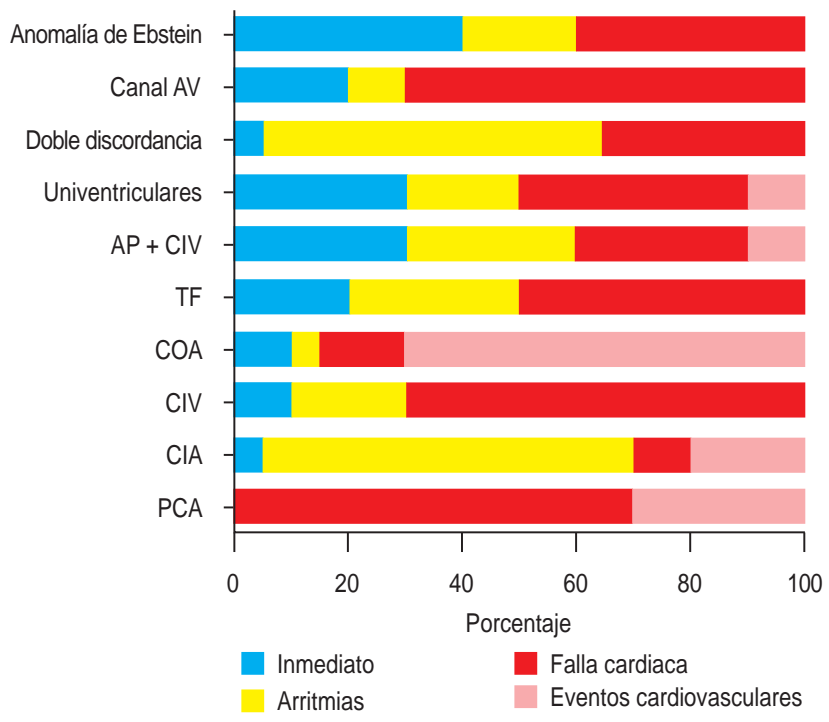

Figura 15.22.2: Causas de mortalidad en cardiopatías congénitas UMAE Hospital de Cardiología del Centro Médico Nacional Siglo XXI. 
entrenamiento del personal de salud. Objetivo: Determinar la supervivencia por tipo de cardiopatía de los pacientes atendidos en una clínica de transición de CC y sus principales causas de muerte. Metodología: Estudio de cohorte de los pacientes atendidos y seguidos de 2013-2014 reparados de CC. Se incluyeron a partir de haber sido reparados y fueron seguidos hasta 2019 o defunción. Fueron registradas todas las CC y los desenlaces fueron atribuidos al procedimiento quirúrgico, arritmias, falla cardiaca y eventos cardiovasculares. Análisis estadístico univariado por curvas de Kaplan Meier. Resultados: La cohorte se integró por 6,572 pacientes, $56 \%$ fueron mujeres, la mediana de edad fue de siete (4-25 años). Del total 36\% fueron adultos (Figura 15.22.1). La supervivencia global a 40 años fue de $74 \%$. En $27 \%$ de las mujeres se registró embarazo exitoso. La anomalía de Ebstein y las cardiopatías univentriculares fueron las de menor supervivencia. La incidencia acumulada de desenlaces fue de siete de cada 100 pacientes y la densidad de incidencia fue de 32 pacientes por cada 10 años. La principal causa de muerte fue la falla cardiaca (Figura 15.22.2). Discusión y conclusiones: La supervivencia en esta cohorte es menor debido al retraso en la atención y la interrupción de los tratamientos. Es necesario la formación de más recursos humanos. 